\title{
Small Animal X-ray Computed Tomography
}

\author{
Cristian T. Badea
}

\section{CONTENTS}

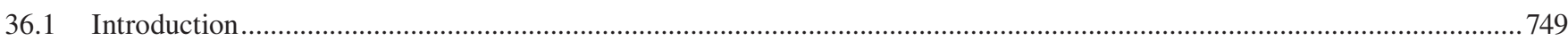

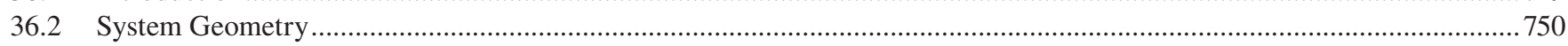

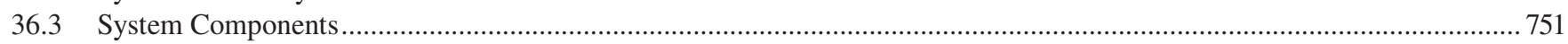

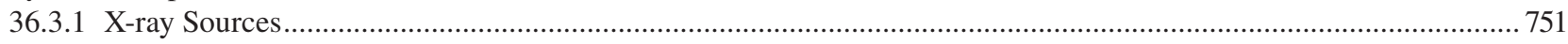

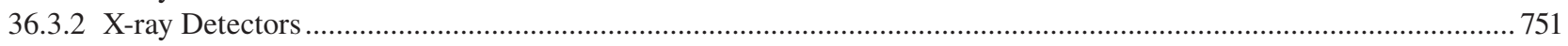

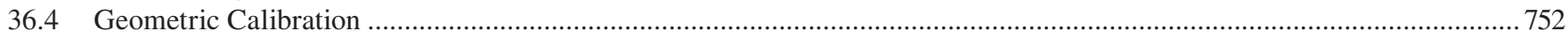

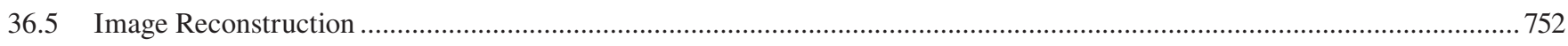

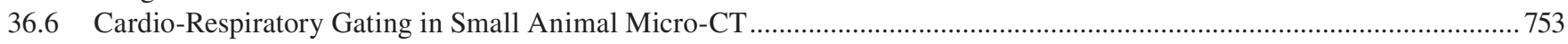

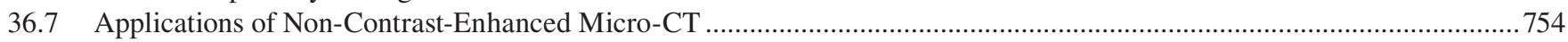

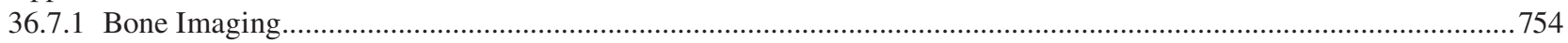

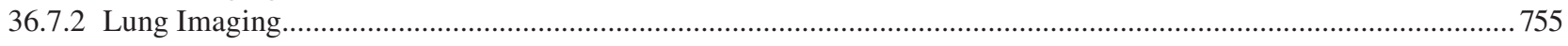

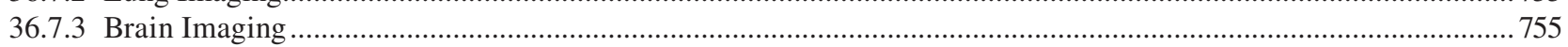

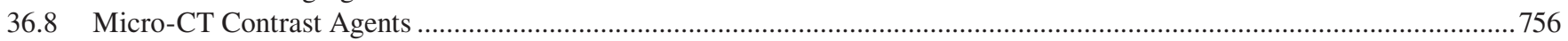

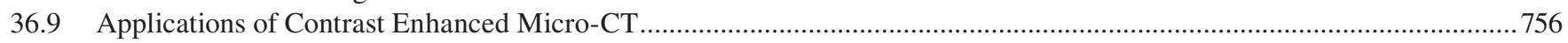

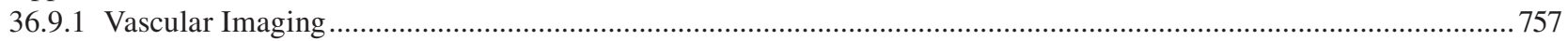

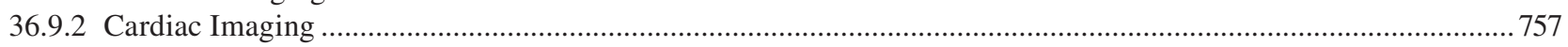

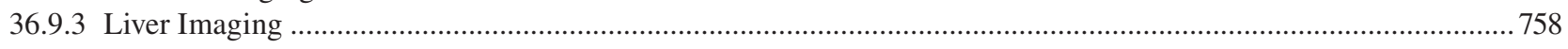

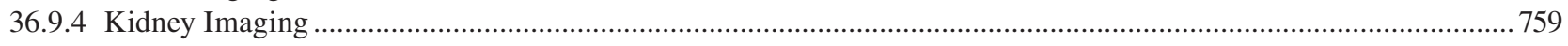

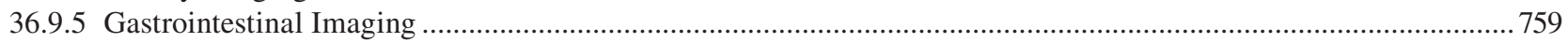

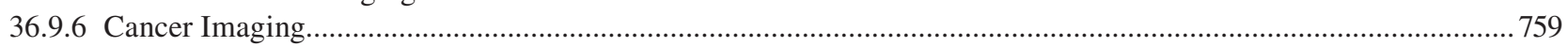

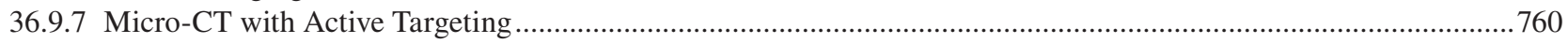

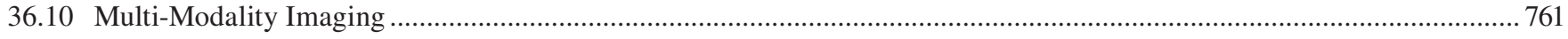

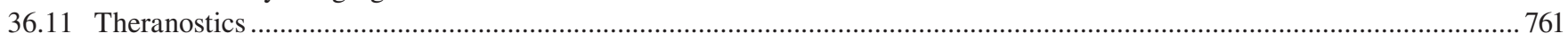

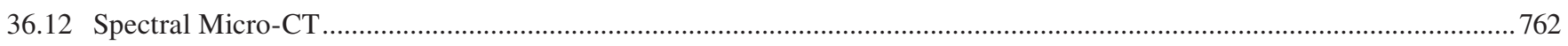

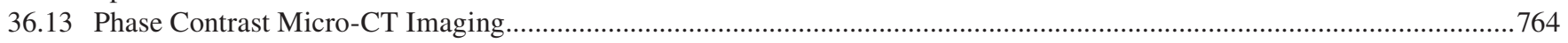

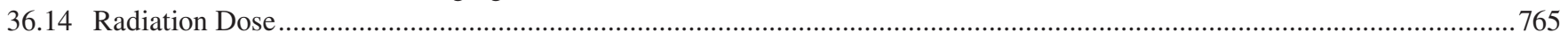

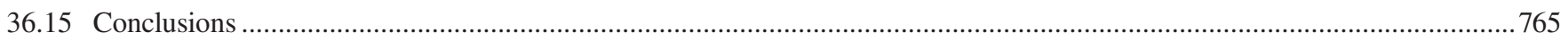

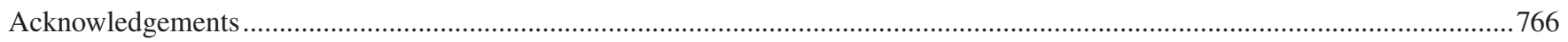

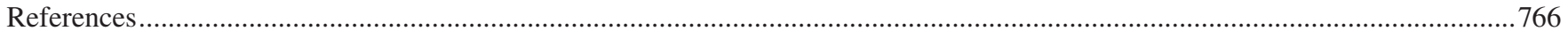

\subsection{Introduction}

X-ray computed tomography (CT) systems with high resolution (also known as micro-CT systems) have been developed over the last two decades and have been used with great success in small animal studies. A micro-CT scanner is based on the same underlying physical principle as a clinical CT scanner, but it is designed for higher-resolution imaging. It produces three-dimensional (3D) tomographic data at microscopic resolution (voxel size $\left.\leq 100 \mu \mathrm{m}^{3}\right)$ by taking hundreds of $2 \mathrm{D}$ projections from multiple angles around the animal (Holdsworth and Thornton 2002). The X-ray source produces a cone beam, which is projected through the specimen with the resultant radiographic density of the specimen projected onto a $2 \mathrm{D}$ detector. The projections are used by a reconstruction algorithm that is generally based on the filtered backprojection (Kak and Slaney 1988) (see Figure 36.1). The resultant micro-CT scan is a 3D matrix of voxels with values proportional to the mean linear attenuation coefficient of the material within each voxel; the $3 \mathrm{D}$ matrix can be sliced in any orientation to reveal different views of the anatomy.

There are many commercially available micro-CT scanners for in vivo and ex vivo imaging. In vivo scanners are used to scan live animals (mostly mice and rats), while ex vivo scanners are used for imaging dead specimens and materials, such as bone and scaffolds. There are major differences between the two types of scanners.

With in vivo micro-CT instruments, the animal remains alive and available for longitudinal studies, thus reducing the number 

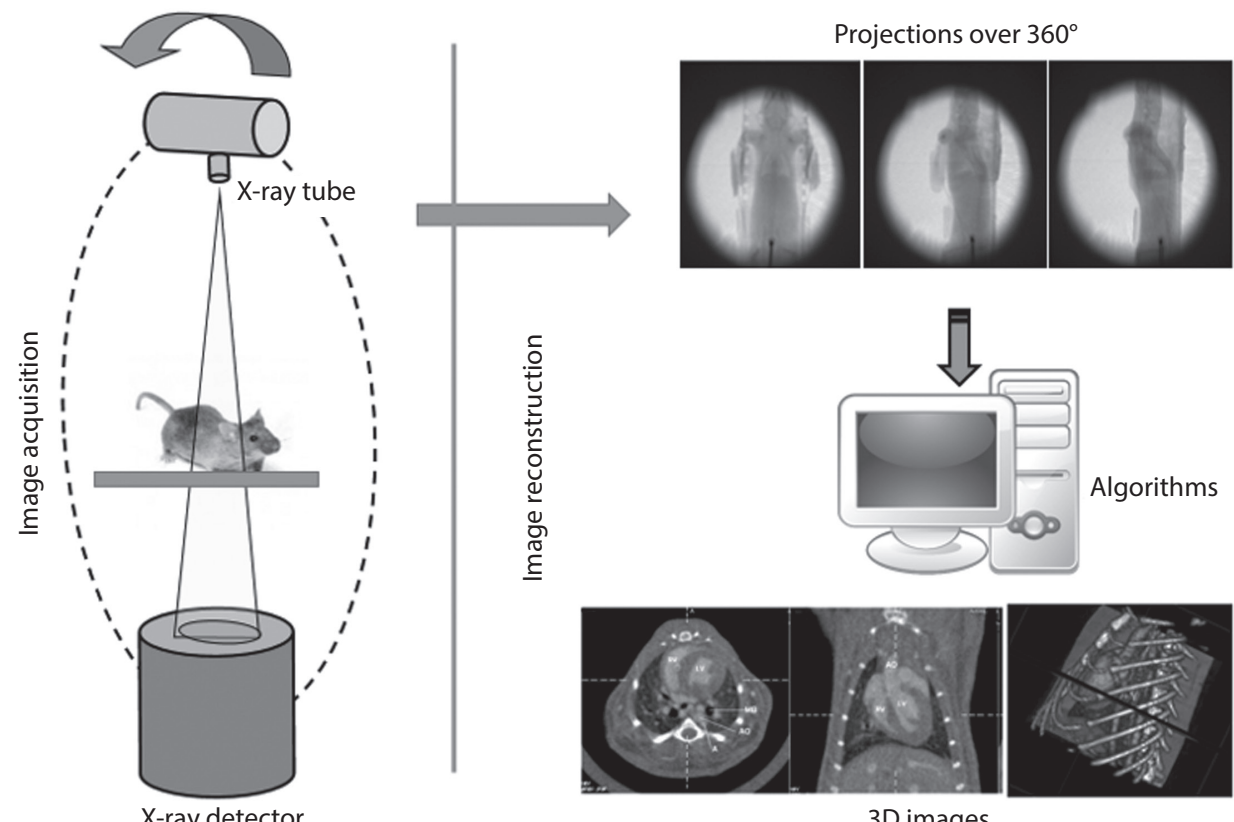

3D images

FIGURE 36.1 The micro-computed tomography (micro-CT) imaging process involves acquiring cone-beam projections with a full rotation of an X-ray source and detector around the animal or specimen. Next, the projections are used by a reconstruction algorithm to create 3D tomographic images.

of animals required. Longitudinal studies can be performed to assess, for example, the effects of diet, drugs, and various treatments on tumors, or bone growth. In vivo micro-CT data are currently also used as a reference for registration with many micro-PET (positron emission tomography) or micro-SPECT (single photon emission computed tomography) scans. For in vivo systems, it is best to have the animal remain stationary during the scan, with its biological measures (e.g. electrocardiogram [ECG], breathing, temperature) monitored. A heater is used to keep the animal warm during the procedure.

Ex vivo micro-CT instruments typically handle applications such as imaging specific organs of an animal that are excised (such as bone, tumors), biomaterial studies, implants, or material studies. For ex vivo micro-CT scanners, physiological motion is not a concern, so monitoring is not needed. This means that the specimen stage can rotate while the X-ray source and detector are stationary. From an imaging performance standpoint, ex vivo micro-CT instruments allow for higher spatial resolution, better signal-to-noise ratios, and therefore better images compared to in vivo imaging. However, this better imaging performance requires longer scan times and higher dose (since dose to the sample is not an issue for ex vivo scanning).

Consequently, there are advantages to both in vivo and ex vivo micro-CT systems and they can be used as complementary systems, depending on the application.

This chapter provides an overview of these aspects of microCT: system components, methods, and applications of micro-CT for small animal studies.

\subsection{System Geometry}

There are two possible system design geometries in micro-CT imaging: (1) rotating gantry (tube and detector) and (2) rotating specimen (see Figures 36.2a and b). All of the current commercial systems for in vivo scanning use the rotating gantry geometry, that is, they are scaled versions of the clinical CT scanners (see Figure 36.2a). Using this design, the X-ray tube and detector, which are mounted on a gantry, rotate around an axis, while the (a)

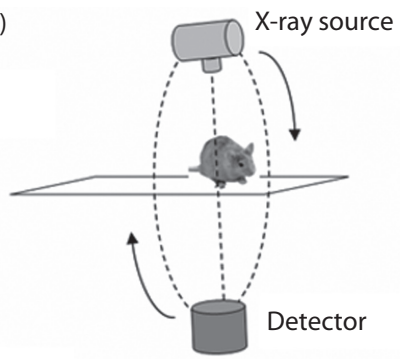

(c)

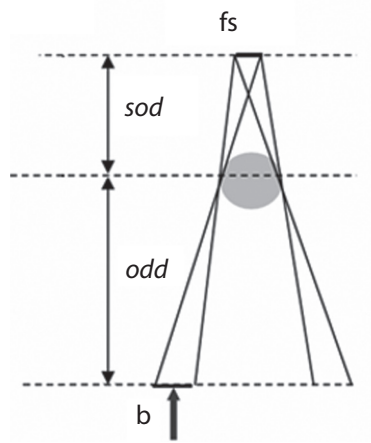

(b)

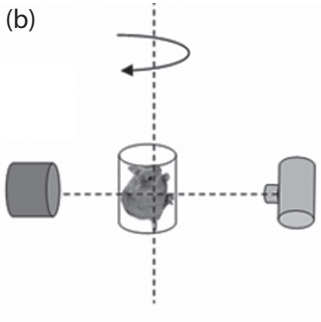

(d)

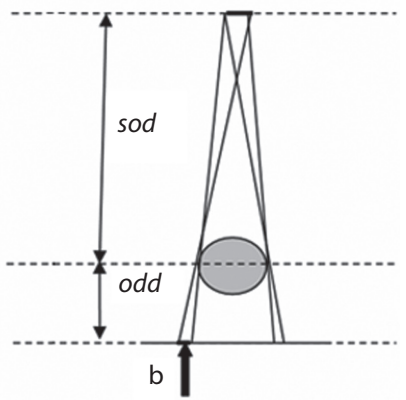

FIGURE 36.2 The two possible design geometries for micro-computed tomography (micro-CT) are based on a rotating gantry (a) or rotating specimen (b). The effect of penumbra blurring: for the same focal spot size (fs) of the X-ray source, the penumbra blurring $b$ is larger when the object is closer to the source (c) than when the object is closer to the detector (d). sod (source-to-object-distance) and odd (object-to-detector-distance). 
animal lies stationary on a table between the tube and detector. For most of these systems, the source-to-object distance (sod) is comparable to the object-to-detector distance $(o d d)$. A geometrical representation of these distances is shown in Figures $36.2 \mathrm{c}$ and $\mathrm{d}$. Positioning the object closer to the X-ray source and farther from the detector allows for an increase in magnification, defined as $M=1+($ oddl/sod $)$, which in turn allows for an increase in resolution (provided that the system is based on the use of a small focal spot X-ray source in order to compensate for the penumbra blurring effect). The penumbra blurring describes the unsharpness produced by X-ray tube focal spots.

The resolution limit due to penumbral blurring $(b)$ in the projection plane is related to the focal spot diameter $(f s)$, odd, and sod by Equation 36.1:

$$
b=\frac{o d d}{\operatorname{sod}} f s
$$

Figure 36.2 shows graphically what happens when the scanning is performed with the object closer to the source (Figure $36.2 \mathrm{c}$ ) or to the detector (Figure 36.2d). According to Equation 36.1 , if odd and sod are comparable, as in most of the rotating gantry systems, the penumbra blurring $b$ becomes comparable or larger than the size of the focal spot and could compromise the spatial resolution if the system uses a large focal spot tube. In the case of systems with a rotating specimen, used in most ex vivo scanners (see Figure $36.1 \mathrm{~b}$ ), the $\mathrm{X}$-ray source and detector are stationary. Such system design is more flexible in changing the magnification by changing the position of the specimen. The specimen could also be placed closer to the detector, that is, odd $<\operatorname{sod}$, resulting in the reduction of penumbra blurring to less than the focal spot size. In this configuration, larger focal spot tubes that provide greater X-ray fluence, and therefore enable shorter exposure times, can be used. Despite these benefits of the rotating specimen design, this type of system has not gained popularity for in vivo imaging, primarily because the animal must be mounted in the vertical position. Combining the benefits of an object magnification and rotating gantry design, some commercial systems with odd $<\operatorname{sod}(M=1.3)$ have also become available (Ross et al. 2006, Du et al. 2007) to allow rapid dynamic micro-CT, albeit at the price of reduced resolution.

\subsection{System Components}

\subsubsection{X-ray Sources}

The choice of the X-ray source strongly affects micro-CT system performance. Due to the tradeoff between focal spot size and thermal loading of the source's metallic anode, most X-ray tubes with micro-focus tubes (focal spot diameter: $<\sim 50 \mu \mathrm{m}$ ) operate with very low photon output (in the order of 100-times lower) compared to the high-power tubes used in clinical scanners (Badea et al. 2004b). This fact explains the increase in the average scan time required in micro-CT compared with clinical scans.

The most-used X-ray sources for micro-CT are micro-focus, fixed tungsten anode tubes operating in continuous mode, with voltages in the range of $20-100 \mathrm{kV}$ and anode currents in the range of $50-1000 \mu \mathrm{A}$. In vivo micro-CT systems working at low magnification often use pulsed X-ray sources with higher power and wider focal spots, in the range of $0.3-0.6 \mathrm{~mm}$. The use of pulsed X-ray sources is especially important for prospective ECG gating (Badea et al. 2008b). This type of imaging can also be done with sources operating in continuous mode by using external shutters.

For ex vivo imaging of small biological samples at very high magnification, the need for very small focus is more important because it represents a major influencing factor of the spatial resolution. Reflection-anode X-ray tubes with focal spots in the range of 5-20 microns are available on the market. Because of the very high power density that such narrow electron beams can release on the target surface, the anode current must be controlled as a function of focal spot size to prevent melting (Grider and Erway 1986). The maximum anode currents in most microfocus X-ray tubes are in the order of $100-200 \mu \mathrm{A}$, with maximum power $<10 \mathrm{~W}$. Other drawbacks of reflection-type X-ray sources with thick targets include a relatively narrow aperture of the cone beam (in the range of $30-60^{\circ}$ ) and a limited capability of reducing the focus-to-object distance below a few millimeters. Non-uniformity of the beam intensity due to the heel effect can be reduced by flat-field (gain) correction; however, accounting for the variation in the X-ray spectrum across the beam profile is difficult (Fivez et al. 1996). Open-type sources with thin-target transmission anodes overcome these limitations, providing radiation beams with angular apertures in the range of $140-160^{\circ}$ with the possibility of putting the object virtually in contact with the focus. However, the back of transmission targets cannot be cooled with liquid, reducing the maximum possible power per unit area compared to reflection anodes. The spatial resolution obtainable with this type of source can be $<1$ micron, which is similar to synchrotron-based imaging. Nevertheless, synchrotron-based micro-CT and nano-CT are still superior to laboratory-level micro-CT and nano-CT systems in terms of contrast resolution and signal-to-noise ratio because synchrotrons provide tunable, monochromatic radiation ${ }_{k}$ avoiding beam hardening artifacts, and much higher photon fluxes, reducing noise (Ritman et al. 1997, Brunke et al. 2008, Stolz et al. 2011).

Other X-ray tube designs have been proposed. Cao et al. have investigated the use of a compact field-emission micro-focus X-ray source based on carbon nanotubes (Cao et al. 2009, 2010). In this type of source, the metal filament cathode is replaced by a field-emission cathode that is capable of emitting electrons at room temperature with voltage controlled output current (Yue et al. 2002).

\subsubsection{X-ray Detectors}

There are two categories of micro-CT X-ray detectors: (1) energy-integrating and (2) energy-discriminating detectors. The majority of detectors used in micro-CT scanners are energyintegrating. Most micro-CT systems to date employ digital flatsurface 2D detectors leading to a cone-beam scanning geometry. Early prototype micro-CT systems employed X-ray image intensifiers read by charge-coupled devices (CCDs) (Feldkamp et al. 1989, Boone et al. 1993, Holdsworth et al. 1993). Later, in the mid-nineties, combined detection systems made up of scintillator screens coupled to CCDs via fiber-optic bundles, with various 
de-magnifying ratios, became the standard for micro-CT imaging (Paulus et al. 2000, Goertzen et al. 2004).

More recently, advances in complementary metal oxide semiconductor (CMOS) technology led to the production of large area detectors with high frame rates, which are the most widely used systems for in vivo, small animal imaging (Lee et al. 2003, Kalender and Kyriakou 2007). Cooled CCDs are still in use, especially in applications involving low X-ray fluences, due to their very low dark noise with respect to flat-panel CMOS detectors. Also reported in the literature are studies on the use of direct conversion detectors coupled to thin-film transistor (TFT) arrays (Goertzen et al. 2004) and of single-pixel or small area detectors operating in photon counting mode (Paulus et al. 2000, Panetta et al. 2007, Roessl et al. 2011) for small animal micro-CT.

The energy discriminating photon-counting X-ray detectors (PCXD) are the subject of intensive research and promise to make spectral CT a reality. In fact, micro-CT is the testing stage for spectral CT using PCXD (Taguchi and Iwanczyk 2013). PCXDs with energy binning can improve CT performance by counting and binning each X-ray detected into a number of energy bins equal to the number of energy thresholds per pixel. PCXDs allow for the elimination of dark noise in the image by rejecting all counts below the signal and also allow for spectral separation. The technology, currently in its infancy, is set to grow rapidly. Sensor materials for PCXDs include silicon $(\mathrm{Si})$, gallium arsenide (GaAs), and cadmium telluride (CdTe) with pixel sizes as small as $55 \mu \mathrm{m}$ (Taguchi and Iwanczyk 2013). Yet, there remain technical limitations to spectral detectors that preclude their immediate replacement of conventional, energy-integrating detectors for biomedical CT applications (Taguchi et al. 2011). For example, $\mathrm{Si}$ is an X-ray detector material with fast signal collection and low susceptibility to signal pile-up, but suffers from a high fraction of Compton interaction at X-ray energies encountered in CT practice, degrading its spectral imaging capabilities (Bornefalk and Danielsson 2010). Among the best available PCXDs, the Medipix detectors have a small pixel size and are best suited for imaging of small animals in preclinical applications (Yu et al. 2012, Taguchi and Iwanczyk 2013). Medipix is a series of photon-counting detectors for X-ray micro-imaging from the Conseil Européen pour la Recherche Nucléaire (CERN) (Campbell et al. 1998). There are already multiple generations of this PCXD. Medipix1 had a pixel size of $170 \times 170 \mu \mathrm{m}$. Medipix 2 reduced the pixel size to $55 \times 55 \mu \mathrm{m}$. The performance of Medipix2 was limited by charge-sharing over neighboring pixels, compromising energy resolution. Medipix3 addressed this problem using a photon-processing chip with special circuitry to allow charge deposition in adjacent pixels to be summed and analyzed with two simultaneous energy thresholds and without spectral distortion (Yu et al. 2012). The readout logic supports eight energy thresholds over $110 \times 110 \mu \mathrm{m}$ for spectroscopic imaging. A commercially available micro-CT system (MARS; University of Canterbury, Christchurch, New Zealand) is equipped with Medipix 3 detectors. We review spectral microCT in separate Section 36.12.

The projection raw data from the X-ray detector are first preprocessed to compensate for the imperfectness of the system, for example, detector response heterogeneity, X-ray flux intensity drift, defective pixel correction, dark current subtraction, and mechanical geometry calibration.

\subsection{Geometric Calibration}

Successful micro-CT imaging is conditioned by an accurate knowledge or calibration of the sampling geometry, also known as alignment. Due to requirements of very high resolution for micro-CT, the allowed mechanical tolerances in geometric variables such as the source-to-detector distance, source-to-object distance, the tilting angles of the detectors, or axis of rotation, are on the same order of magnitude of the thermal drifts of the components (Parkinson and Sasov 2008). An uncorrected displacement of the axis of rotation of a tenth of the detector pitch may result in a significant image blurring, and consequently, in a loss of image spatial resolution. Many methods have been proposed for the measurement of the misalignment parameters from the analysis of the acquisition data in cone-beam geometry (Noo et al. 2000, Beque et al. 2003, von Smekal et al. 2004, Yang et al. 2006, Defrise et al. 2008). Such parameters can be embedded into the reconstruction process via a projection matrix in order to produce misalignment free reconstructed images (Karolczak et al. 2001).

Most geometric calibration methods make use of a phantom consisting of two or more small spherical objects, such as metallic beads; each bead traces an ellipse on the detector plane during the tomographic acquisition, hence the geometry of the tomographic system can be determined by the analysis of all the ellipses.

Despite their accuracy, a drawback of the calibration methods based on special phantoms is that they need dedicated acquisition sessions to be applied. Many commercial systems for micro-CT imaging now provide zoom-in capability and variable geometry. In such cases, nearly continuous calibration of the system's geometry is necessary to guarantee the stability of the image spatial resolution. For this purpose, methods of selfcalibration have also been developed that attempt to estimate a subset of the geometric parameters of the system from the projection data of a generic object (Panetta et al. 2008) or by postreconstruction optimization of image-based metrics of image sharpness (Wicklein et al. 2012).

\subsection{Image Reconstruction}

All CT image reconstruction algorithms solve an inverse problem that maps cone-beam projection data to a 3D tomographic representation of the specimen (Paulus et al. 2001).

There are two major types of algorithms: (1) filtered back projection (FBP)-based algorithms, and (2) iterative algorithms, (Kak and Slaney 1988). The most commonly used reconstruction algorithms in CT and micro-CT are based on FBP. In FBP, the acquired, 2D, cone-beam projections are filtered, using a convolution kernel that reduces the blurring inherent to the back projection process, and then they are back projected (i.e., smeared back) through the object space at the appropriate angle to generate an image. While first developed for 2D imaging (parallel or fan beam), convolution back projection was adapted by Feldkamp (Feldkamp et al. 1984) to compensate for the conebeam geometry. This method forms the basis for the reconstruction algorithms used in most current micro-CT systems. While Feldkamp's algorithm is considered to be an approximation, 
(a)
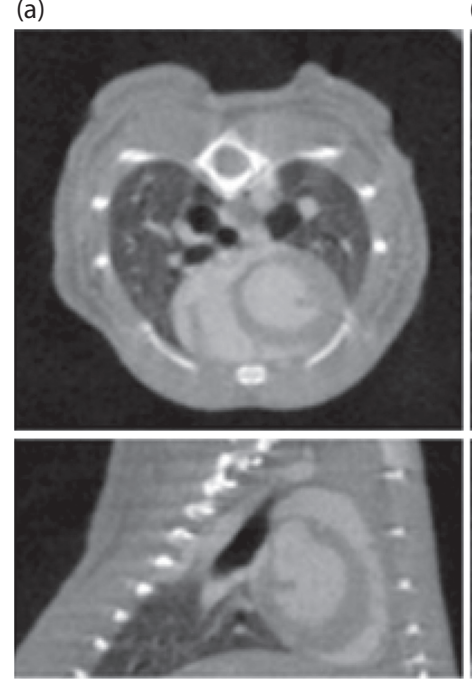

(b)
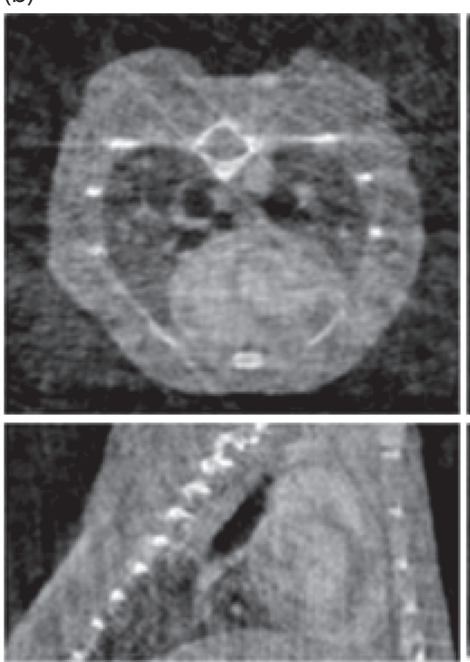

(c)

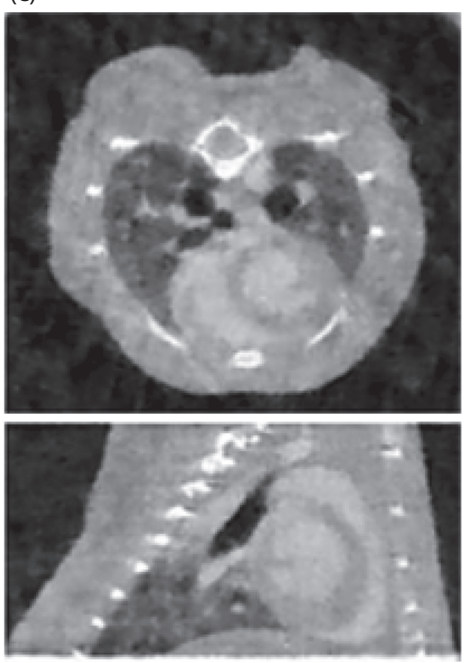

FIGURE 36.3 In vivo cardiac micro-computed tomography (micro-CT) images reconstructed from prospectively (a) and retrospectively (b) gated 3D cone-beam measurements. In (a) filtered back projection reconstruction was used with 380 prospectively gated projections, while (b) and (c) show results for using 95 retrospectively gated projections with filtered back projection reconstruction and total variation (TV)-CT reconstruction, respectively.

since the circular sampling trajectory does not satisfy Tuy's data sufficiency condition (Tuy 1983), the quality of the reconstructed images is acceptable if the cone angle $<10^{\circ}$ (Holdsworth and Thornton 2002), except for very unusual object configurations (Defrise and Clack 1995).

When the number of projections available for reconstruction is limited and/or the projections are very noisy (e.g., low dose scanning), both iterative and statistical reconstruction algorithms can provide notably better image quality than FBP. Reduction in the number of views translates directly to reduced radiation dose to the animal. Furthermore, it is sometimes not possible to acquire projections with regular-angular sampling in dynamic imaging applications such as cardiac studies. In such cases, the reconstruction is affected by artifacts when FBP is used. Iterative algorithms, such as the algebraic reconstruction technique (ART) (Gordon et al. 1970), present an alternative to FBP with the potential for robust reconstruction given less than ideal projection data (Kak and Slaney 1988). With such algorithms, the reconstructed volume is refined by repeatedly comparing simulated re-projections of the reconstructed volume with the original projections.

Artifacts from FBP reconstruction with an irregular-angular distribution of projections manifest as long thin streaks and shading artifacts. Total variation (TV) regularization has been recognized for its ability to suppress artifacts, while leaving boundaries between homogeneous regions intact (Rudin et al. 1992). TV regularization can be interleaved with iterative reconstruction algorithms such as the simultaneous algebraic reconstruction technique (SART) by performing one or more iterations of each in turn. To illustrate the power of SART-TVbased reconstruction using irregularly sampled projections, we present its application in 4D cardiac micro-CT. Specifically, Figure 36.3 compares image quality with FBP reconstruction for a well sampled case in which projections are acquired prospectively gated on ECG with a regular angular distribution (Figure 36.3a) and with a limited number (only 95), and irregular angular distribution of projections corresponding to a retrospective-gated acquisition (Figure 36.3b). The artifacts associated with the FBP reconstruction with retrospective gating are clearly visible in Figure 36.3b. With an iterative reconstruction such as SART-TV, the artifacts are alleviated (Figure 36.3c) (Song et al. 2007).

Statistical image reconstruction is another class of iterative reconstruction algorithms, first introduced for transmission imaging in nuclear medicine, but also applied for CT reconstruction (Elbakri and Fessler 2002). In essence, these algorithms treat the reconstruction as a statistical estimation problem and have the advantage that they can accurately model Poisson noise in the projection data. This results in lower noise levels with statistical reconstruction for the same data when compared with FBP reconstruction.

One major limitation of iterative reconstruction algorithms is their running time. To overcome this limitation, the graphics processing unit (GPU) has been recognized as an alternative computer architecture with potential for accelerating CT reconstruction (Xu and Mueller 2007). This parallel architecture is ideal for CT reconstruction steps in which the same arithmetic operations must be performed at multiple pixels or voxels (e.g. projection, back projection). The development of programming tools for general-purpose computing on the GPU, such as NVIDIA's Compute Unified Device Architecture (CUDA, Santa Clara, CA), has facilitated the acceleration of $\mathrm{CT}$ reconstruction, presenting the opportunity to explore more complex iterative algorithms. Micro-CT is already benefiting from GPU-based implementations of analytical, iterative, and statistical image reconstruction (Zhao et al. 2007, Jakab et al. 2009, Johnston et al. 2010a).

\subsection{Cardio-Respiratory Gating in Small Animal Micro-CT}

The influence of cardiac and respiratory motion during in vivo scanning can be minimized by using cardio-respiratory gating. 4D micro-CT used in cardio-pulmonary studies typically employs either prospective gating (PG) or retrospective gating (RG). In PG, acquisition is triggered by the coincidence of a selected respiratory 
phase and a selected cardiac phase. This produces a set of projections with a constant angular step, resulting in reconstructed images that are free of streaking artifacts. However, because of the time spent waiting for the coincidence of cardiac and respiratory events, the scan time can take as long as one hour to cover 10 different phases of the cardiac cycle (Badea et al. 2004b).

In $\mathrm{RG}$, the projection images are acquired at a rapid and constant rate without waiting for cardiac and respiratory coincidence. Respiratory and ECG signals are monitored and saved in synchrony with the acquisition of the projections. Using these signals post-sampling (i.e., retrospectively), the projections are sorted into different subsets corresponding to different cardiac and respiratory phases. With this protocol, the scan time can be shortened to 50 seconds when using a slip ring gantry (Drangova et al. 2007). However, the irregular angular distribution causes streaking artifacts in the FBP-based reconstructed images. As shown by Figure 36.3, superior results are possible using regularized iterative algorithms such as SART-TV (Song et al. 2007). A different gating strategy called fast prospective gating (FPG) has been introduced (Guo et al. 2012a), which combines the regular angular distribution of PG with the fast scan time of RG. In FPG, multiple projections are acquired at the same angle, corresponding to all cardiac or respiratory phases to be reconstructed, before the cradle is rotated to the next angle. FPG requires onthe-fly computation of the triggering events, which are delayed from the peaks of the respiratory or cardiac signals.

In terms of implementation, most of the proposed methods involve extrinsic cardio-respiratory gating, in which the cardiac and respiratory signals are acquired with dedicated monitoring devices (i.e., ECG leads and a pneumatic respiratory pillow). In addition to these extrinsic gating techniques, an intrinsic imagebased gating approach without any external devices was developed, initially for a clinical, spiral cone-beam CT scanner (Kachelriess et al. 2002). In intrinsic gating, a post-processing algorithm evaluates the center of mass of certain regions of interest (ROIs) within each projection to detect respiratory and cardiac motion (Bartling et al. 2008, Dinkel et al. 2008, Sawall et al. 2011).

\subsection{Applications of Non-Contrast- Enhanced Micro-CT}

Micro-CT images only demonstrate high contrast when there are large differences between material densities (Compton scattering) or atomic weight (photoelectric effect) within the subject. In the case of soft tissue imaging, there is very little natural contrast, and an exogenous high atomic weight contrast agent must be administered for effective imaging ( $\mathrm{Yu}$ and Watson 1999). However, non-contrast-enhanced micro-CT performs well for bone, lung, or even helps in brain studies, in the absence of exogenous contrast agents.

\subsubsection{Bone Imaging}

Bone imaging was one of the very first common applications of micro-CT for small animal imaging (Feldkamp et al. 1989, Kinney et al. 1995). Micro-CT is well-suited for bone imaging because of the natural contrast between bone and soft tissues, which is due to the higher effective atomic weight of bone. This makes micro-CT extremely valuable for non-invasive, highresolution bone imaging without the need for an exogenous contrast agent. Micro-CT can accurately quantify a variety of bone parameters, including cross-sectional area, cortical thickness, bone mineral density, bone volume, bone surface ratio, and trabecular thickness (Bouxsein et al. 2010). Structural micro-CT studies have examined bone architecture (Waarsing et al. 2005, Hsu et al. 2014), bone remodeling (David et al. 2003, Cowan et al. 2007), and osteoarthritis (Appleton et al. 2007, McErlain et al. 2008). Micro-CT has also been used to monitor bone healing after treatment with basic fibroblast growth factor (Yao et al. 2005), vascular endothelial growth factor gene therapy (Li et al. 2009), or stem cell therapy (Lee et al. 2009). Micro-CT can also be used to longitudinally track bone loss and structural changes following radiation therapy and bone marrow transplantation (Dumas et al. 2009) or after spinal cord injury (Jiang et al. 2006). In the case of osteoporosis, micro-CT measurements have been used to study disease progression after ovariectomy (Laib et al. 2001) or immobilization (Laib et al. 2000). Micro-CT has also been used to study early bone development and growth (Guldberg et al. 2004). Additionally, micro-CT has been used extensively in studies of bone regeneration (Umoh et al. 2009) and bone tissue engineering (Lin et al. 2005, Ho and Hutmacher 2006). In these cases, micro-CT can quantify mineral content, porosity, and connectivity, as well as accurately determine 3D structures. Figure 36.4 illustrates ex vivo micro-CT bone imaging at various resolutions (Bouxsein et al. 2010). Note that structural bone details are lost as the resolution gets worse. This points to the importance of high resolution being required in bone studies. (a)

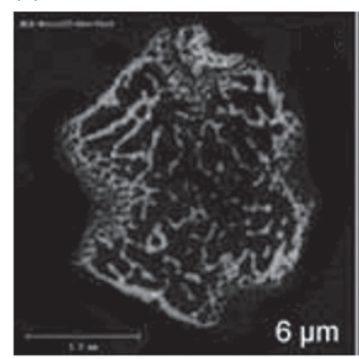

(b)

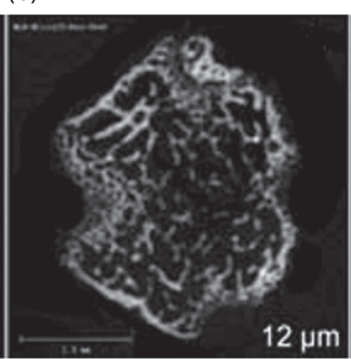

(c)

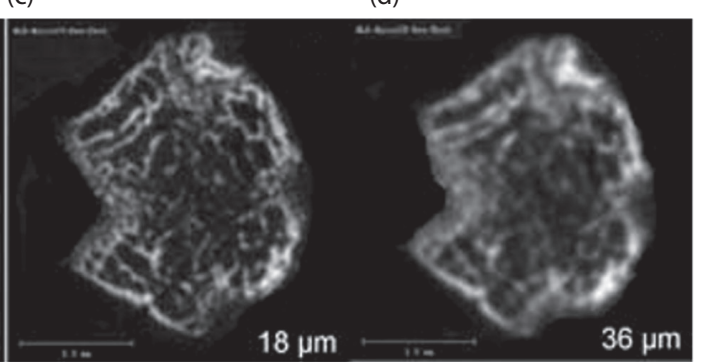

FIGURE 36.4 Effect of voxel size on image quality. 2D gray-scale images of the distal femur of an adult mouse scanned at voxel sizes of (a) $6 \mu$ m, (b) $12 \mu \mathrm{m}$, (c) $18 \mu \mathrm{m}$, and (d) $36 \mu \mathrm{m}$. Images acquired at $70 \mathrm{kVp}, 114 \mathrm{~mA}$, and $200 \mathrm{~ms}$ integration time. (Adapted from Bouxsein, M. L. et al. 2010 . Guidelines for assessment of bone microstructure in rodents using micro-computed tomography. J Bone Miner Res 25(7), 1468-1486. doi:10.1002/jbmr.141.) 


\subsubsection{Lung Imaging}

The large difference in density between air-filled lungs and soft tissues creates high contrast for lung imaging, which also makes CT an extremely useful modality for studying the lung. The primary difficulty in imaging the lungs is respiratory motion. Small-animal breathing rates are three to four times the average respiratory rate for humans, so completing an entire scan between breaths is not practical. Instead, various gating strategies are used, which allow researchers to acquire each projection at the same stage in the respiratory cycle, so that there is only minimal motion from one projection to the next. One of the most effective methods of respiratory gating is to intubate the animal and control the respiration by mechanical ventilation (Hedlund and Johnson 2002, Namati et al. 2006). This allows projections to be acquired at exactly the same point in each respiratory cycle. The $\mathrm{X}$-ray projections can then be acquired automatically at the same point in the measured respiratory cycle (Badea et al. 2004a). For a less invasive approach, the respirations of a freely breathing animal can be monitored using a pressure transducer. This method does not perfectly eliminate respiratory motion, but it is much less invasive than mechanical ventilation and can still resolve features down to $\sim 150$ microns (Namati et al. 2006). Retrospective gating is also possible, in which many projections are acquired rapidly and sorted post-acquisition according to the phase of the respiratory cycle. Subsequently, these sorted projections are used for the reconstruction of tomographic images corresponding to each phase of the respiratory cycle (Ford et al. 2007).

Micro-CT has been used to study a wide variety of lung diseases. Micro-CT can be used to longitudinally monitor mice for the presence of lung metastases ( $\mathrm{Li}$ et al. 2006), as well as follow the growth of lung tumors (Hori et al. 2008, Namati et al. 2010, Li, et al. 2013a, Rudyanto et al. 2013). The treatment efficacy of chemotherapy (Ueno et al. 2012) or radiation therapy (Perez et al. 2009, 2013, Kirsch et al. 2010) on lung tumors can be measured using micro-CT, and lung injury resulting from radiation therapy can also be assessed (Saito and Murase 2012). In addition to tumor characterization, micro-CT is also useful for imaging diseases of the lung parenchyma. Mouse models of emphysema created by intra-tracheal instillation of elastase (Postnov et al. 2005, Artaechevarria et al. 2011, De Langhe et al. 2012, MunozBarrutia et al. 2012) or exposure to cigar smoke (Sasaki et al. 2015) have been developed and characterized by micro-CT. In emphysema, CT values decrease compared to normal lung due to the loss of soft tissue parenchyma and increased air-trapping.

A mouse model of bleomycin-induced lung fibrosis has also been studied extensively by micro-CT (Shofer et al. 2007, Shofer et al. 2008, De Langhe et al. 2012), and this model has been used with micro-CT for the preclinical evaluation of drug efficacy (Scotton et al. 2013, Choi et al. 2014, Zhou et al. 2015). In fibrosis, micro-CT values increase due to an expansion of the parenchyma tissue. Lung compliance and lung volume, which are important factors in both emphysema and fibrosis, can also be measured by micro-CT. Animals are mechanically ventilated at multiple pressures and the lung volume at each pressure is measured. The resulting lung pressure-volume curve can be used to calculate lung compliance (Guerrero et al. 2006, Shofer et al. 2007). Figure 36.5 shows an example of longitudinal lung micro-CT imaging in a control and a mouse with bleomycin-induced fibrosis at one

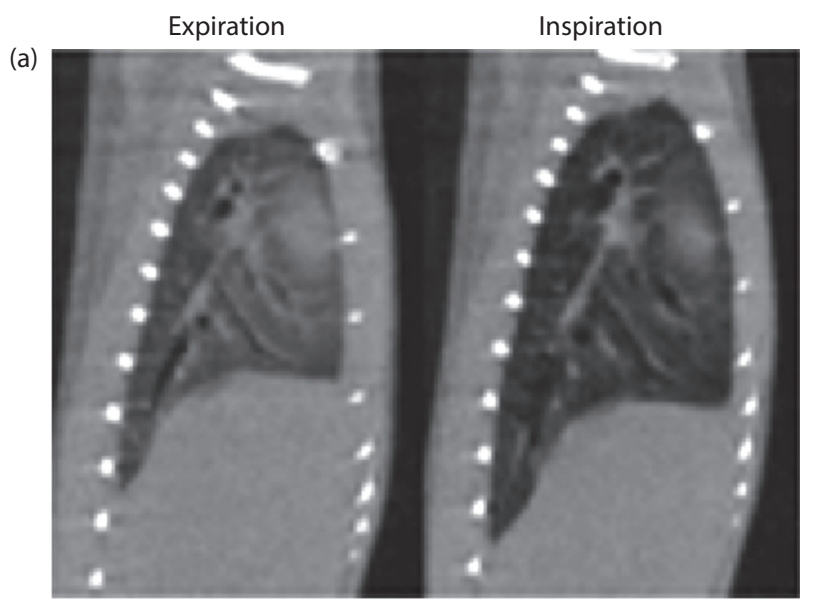

(b)

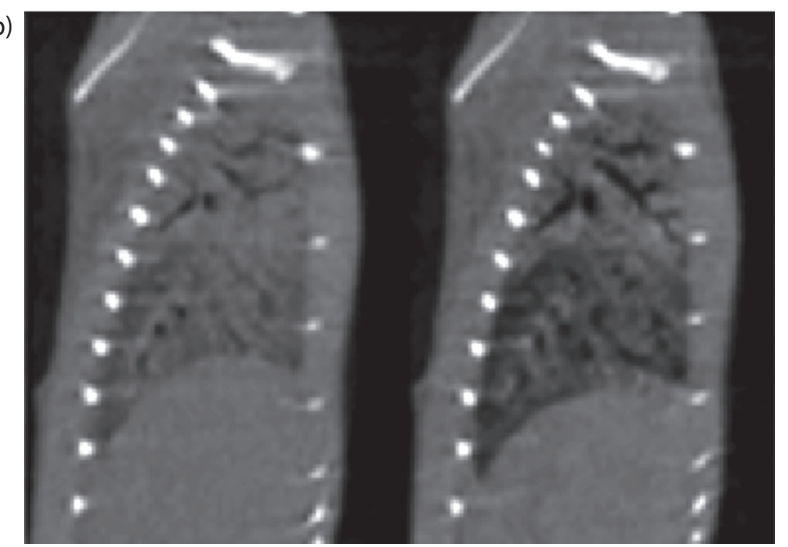

FIGURE 36.5 Representative micro-computed tomography (micro-CT) images at end expiration and end-inspiration of a control mouse (a) and a mouse with fibrosis at week 1 (b). Note darkening of lung parenchyma, diaphragmatic depression, and enlargement of the airways present in the control mouse (a). In week 1 fibrosis mouse (b), there is dense consolidation in the expiratory images with ground-glass opacities at end-inspiration in the peripheral and lower lung fields with persistent consolidation apically. In addition, there is limited movement of the diaphragm, suggesting reduction in lung volumes at end-inspiration.

week post-instillation (Shofer et al. 2008). The micro-CT images were acquired both at end-expiration and end-inspiration using respiratory gating. In the fibrosis mouse, the images show dense consolidation at expiration with ground-glass opacities at inspiration. Furthermore, there is limited movement of the diaphragm, suggesting reduction in lung volumes at end-inspiration.

\subsubsection{Brain Imaging}

A lack of significant contrast often poses a barrier to the imaging of brain disease models with non-contrasted micro-CT. However, since the brain resides in a cavity that is circumscribed by the cranial bones of the skull, micro-CT can become useful. For example, micro-CT has been used to locate metallic electrodes in the brain for neural recording studies (Borg et al. 2015). Simultaneous neural recordings taken from multiple areas of the rodent brain can provide insight about spatially distributed neural circuitry. The traditional methods for localizing the precise location of surgically implanted metallic electrodes into intact rodent brains require slicing and staining of physical brain tissue, and are cumbersome. But, micro-CT localization 
(a)

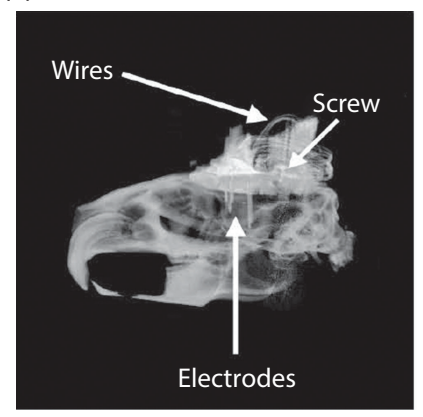

(b)

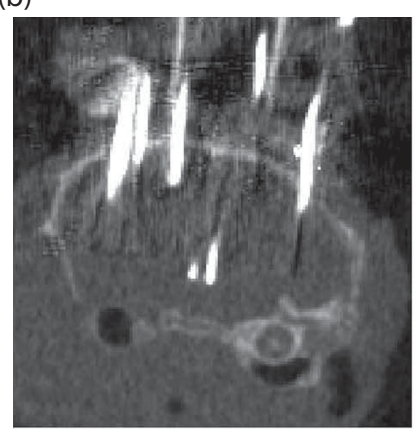

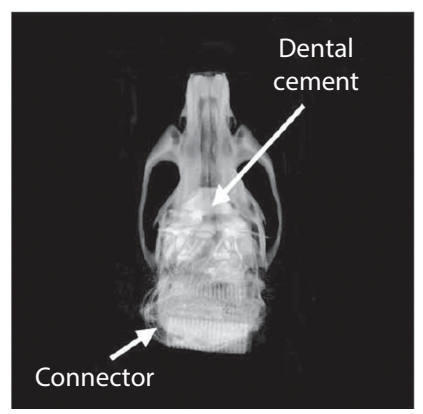

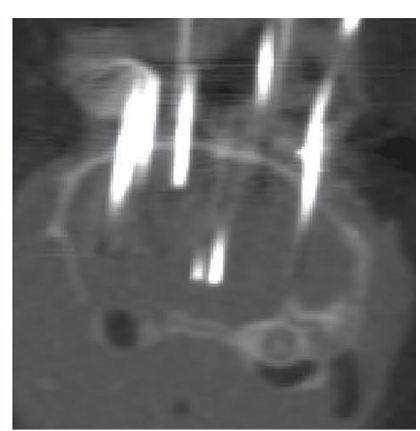

FIGURE 36.6 Micro-computed tomography (micro-CT) images of implanted rat brains. (a) 3D reconstructions illustrate the $3 \mathrm{D}$ structure of electrode bundles and head implants. (b) Examples of CT cross-sections without (left) and with (right) artifact reduction applied. (Reproduced from Borg, J. S. et al. 2015. Eneuro 2(4):ENEURO. 0017-15.2015.)

can provide a better solution. Figure 36.6 presents examples of micro-CT images used for electrode localization. Being metallic, note that the electrodes produce streaking artifacts. Using adequate metallic artifact reduction techniques can make these micro-CT images sufficient for registration to a magnetic resonance imaging (MRI) brain atlas, and thus provide accurate localization of the electrodes in known brain structures.

\subsection{Micro-CT Contrast Agents}

Due to lack of inherent contrast for soft-tissue imaging, the majority of CT scans make use of high atomic weight contrast agents. In current clinical practice, iodine is the most commonly used element for intravascular CT contrast. Iodine contrast agents are made up of water-soluble aromatic iodinated compounds. These compounds provide effective contrast due to their high atomic number, which produces a strong photoelectric effect. Because CT is relatively insensitive to contrast, high concentrations of contrast agent (up to $400 \mathrm{mg}$ iodine $/ \mathrm{mL}$ ) must be injected to produce adequate image enhancement. Clinical CT contrast agents are generally safe, but severe adverse reactions sometimes occur due to their high osmolality and high viscosity (Namasivayam et al. 2006, Tepel et al. 2006, Wang et al. 2007). Iodinated contrast agents are rapidly cleared from the bloodstream by the kidneys (Bourin et al. 1997), so there is only a very short window for imaging after injection. Additionally, these agents quickly distribute from the intravascular to the extravascular space throughout the body. Initially, this provides useful contrast, but after a short time, this nonspecific uptake leads to uniform enhancement throughout most of the body.

For small-animal imaging, the use of clinical contrast agents is particularly difficult. Small animals have much higher renal clearance rates than humans, so injected contrast agents are rapidly excreted. This can be illustrated for a mouse. In the average adult mouse, blood volume is approximately $1.5-2.0 \mathrm{~mL}$ (Diehl et al. 2001), and the glomerular filtration rate (the volume of plasma filtered by the kidneys per time) is approximately $0.4 \mathrm{~mL} / \mathrm{s}$ (Cervenka et al. 1999). Therefore, the whole mouse blood volume is filtered by the kidneys in less than five seconds. Consistent with this filtration rate, it has been shown that clinical iodine contrast agents drop to undetectable levels in the bloodstream within four seconds of injection in a mouse (Lin et al. 2008). This rapid clearance of contrast agent severely limits the useful application of clinical contrast agents in small animals.

To overcome the rapid clearance of traditional contrast agents, blood pool contrast agents have been developed, which exhibit prolonged blood residence time and stable enhancement for minutes to hours. Blood pool agents are made up of a wide variety of high molecular weight compounds or nanoparticles that avoid renal clearance due to their large size. Iodine-based blood pool agents include iodine-containing polymers (Galperin et al. 2007, Aviv et al. 2009), micelles (Trubetskoy et al. 1997, Torchilin et al. 1999), emulsions (de Vries et al. 2010, Hallouard et al. 2013, Li et al. 2013b), and liposomes (Krause et al. 1993, Petersein et al. 1999, Mukundan et al. 2006, Ghaghada et al. 2011). The development and use of these iodine-containing blood pool contrast agents have been reviewed elsewhere (Hallouard et al. 2010, Annapragada et al. 2012, Cormode et al. 2014, Li et al. 2014). Some iodine-containing blood pool agents are commercially available for small animal research, including Fenestra ${ }^{\circledR}$ (MediLumine) and Exia ${ }^{\mathrm{TM}}$ (Binitio Biomedical, Inc.).

Over the past several years, metal nanoparticle contrast agents have been developed, which incorporate a wide variety of elements. The most commonly used metal nanoparticles for microCT consist of gold. Gold nanoparticles produce greater CT enhancement than iodinated contrast agents because of the high atomic number of gold $(Z=79)$ compared to iodine $(Z=53)$. Gold nanoparticles are particularly promising for in vivo imaging applications because gold is extremely inert and gold nanoparticles can be readily modified with surface-linked molecules to render them biocompatible (Li et al. 2012). Surface conjugation is important for nanoparticle contrast agents, because bare nanoparticles adsorb serum proteins and are readily recognized and cleared by the immune system. A variety of molecules can be added to the nanoparticle surface to decrease nanoparticle clearance, but the most common modification strategy is the addition of polyethylene glycol (PEG) (Jokerst et al. 2011). Surface PEGylation and size significantly increases the blood residence time of nanoparticles, which allows them to be used as blood pool contrast agents. Nanoparticles for micro-CT have also been developed using other metals, including silver, barium, gadolinium, bismuth, ytterbium, tantalum, and thorium (Jakhmola et al. 2012).

\subsection{Applications of Contrast Enhanced Micro-CT}

The development of nanoparticle contrast agents has opened the door for many exciting applications in small animal imaging. While imaging applications using low molecular weight contrast 
agents have been limited, blood pool contrast agents have now been used for a wide range of imaging applications. Important modern applications for contrast-enhanced micro-CT in small animals include imaging of the vasculature, heart, liver, abdomen, and tumors. Current research of micro-CT contrast agents is now focused on developing agents with active targeting, multimodal, or theranostic capabilities.

\subsubsection{Vascular Imaging}

Vascular imaging for micro-CT is done primarily using blood pool contrast agents. Micro-CT scan times must be longer than clinical CT scan times due to the requirement for much higher resolution. Higher resolution implies a need for more X-ray flux, which is achieved with longer integration time per projection. Early micro-CT scanners required up to an hour to complete a scan. In these cases, low molecular weight contrast agents could not be used for vascular imaging, as they would be cleared from the bloodstream long before the image acquisition was completed. For current micro-CT scanners, scan times of under a minute are now possible. Using these fast protocols, low molecular weight contrast agents have been successfully used for vascular imaging (Kiessling et al. 2004, Badea et al. 2006, Schambach et al. 2010). However, these contrast agents must be either repeatedly or continuously administered over the course of a scan to achieve a constant level of vascular enhancement. This increases the difficulty of imaging and may significantly increase the injected dose of contrast agent. As an alternative to low molecular weight contrast agents, blood pool contrast agents have been successfully used for a variety of vascular applications, including measurements of vascular morphology, diameter, and branching (Vandeghinste et al. 2011), imaging pulmonary vasculature (Johnson 2007), imaging hepatic vasculature (Chouker et al.
2008), imaging tumor vasculature (Badea et al. 2006, Graham et al. 2008), and measuring vascular permeability (Langheinrich and Ritman 2006). By providing a constant level of enhancement within the vasculature over a prolonged period of time (minutes to hours), these contrast agents simplify the acquisition of vascular images using micro-CT and allow for a wider range of imaging protocols to be used. Figure 36.7 shows examples of vascular imaging both in vivo and ex vivo. In Figure 36.7a, micro-CT was used with a liposomal iodine contrast agent in order to study the vasculature associated with primary soft tissue sarcomas of the hindlimb (Moding et al. 2013). Maximum intensity projection is used to render the vascular component. In Figure $36.7 \mathrm{~b}$, we present an ex vivo case. The mouse lungs were perfused with Microfil medium mixture through the pulmonary artery at $3 \mathrm{~mL} / \mathrm{min}$. The study was focused on investigating how beta-arrestins regulate signaling by bone morphogenetic protein type II receptor in pulmonary arterial hypertension (Rajagopal et al. 2011).

\subsubsection{Cardiac Imaging}

Cardiac imaging is challenging in small animals due to their rapid heart rate ( $\sim 600 \mathrm{bpm}$ for mice). Like respiratory gating (see Section 36.6), cardiac gating can be used to minimize artifacts due to cardiac motion in the resulting $\mathrm{CT}$ images. Cardiac gating can be performed either prospectively (Badea et al. 2005, 2008a, 2011b, Ford et al. 2005, Guo et al. 2012a) or retrospectively (Bartling et al. 2007, Song et al. 2007, Badea, et al. 2008b,c, Badea et al. 2011c, Ashton et al. 2014a). In both cases, the ECG of the animal is continuously monitored. In prospective gating, each projection is triggered at a pre-defined point of the cardiac cycle, so that the heart is in the same position in each of the projections. In retrospective gating, projections are acquired rapidly over (a)

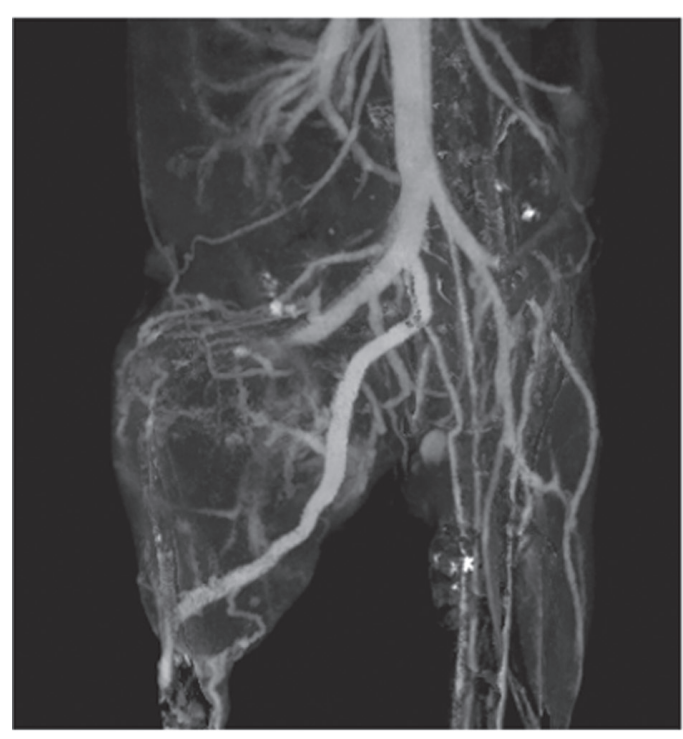

(b)

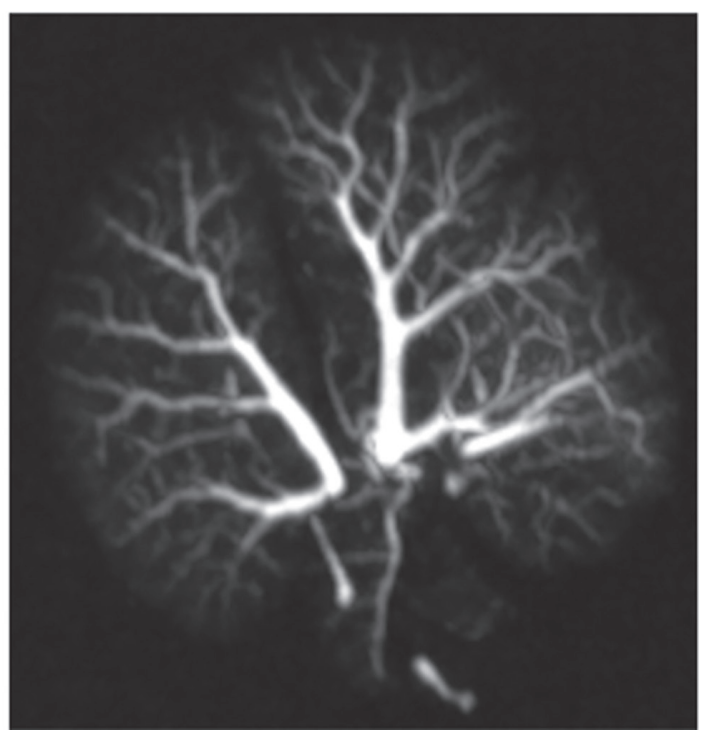

FIGURE 36.7 (a) Coronal maximum intensity projection of the vasculature of a mouse with a soft-tissue sarcoma in the right hindlimb. Micro-computed tomography (micro-CT) imaging ( $88 \mu \mathrm{m}$ voxel size) was performed immediately after injection of a liposomal iodine contrast agent. Bones have been removed from the image by segmentation in order to better visualize the vasculature feeding the tumors. (b) Mouse lungs were perfused with Microfil medium mixture through the pulmonary artery at $3 \mathrm{~mL} / \mathrm{min}$. Beta-arrestins regulate signaling by bone morphogenetic protein type II receptor in pulmonary arterial hypertension. 
several rotations and then the timing of the images is compared to the ECG signals. Each of the projections is next sorted according to its cardiac phase and used for tomographic reconstruction. Retrospective gating is much more rapid but produces an irregular angular distribution of projections, which can cause artifacts during the reconstruction process. Because prospectively gated images are acquired using temporal delays computed based on the ECG signal, they require several minutes to perform. Many cardiac imaging protocols incorporate both respiratory and cardiac gating to minimize overall thoracic motion during the scan (Badea et al. 2004a). We note that intrinsic retrospective gating can also be implemented with cardiac and respiratory motion signals derived from information within each of the acquired projections using image processing, thus avoiding the complications of having ECG or respiratory sensors attached to the mouse (Bartling et al. 2008, Kuntz et al. 2010, Johnston et al. 2010b).

For all cardiac imaging, contrast agents are necessary to differentiate the myocardium from the heart lumen. Because cardiac-gated scans can require several minutes to perform, enhancement of the blood within the heart must remain constant for a prolonged period of time to produce high-quality scans. Such imaging is possible with low molecular weight contrast agents by using continuous infusion during the scan or repeated injections (Sawall et al. 2012), but the vast majority of studies have made use of blood pool contrast agents. Because images can be acquired over multiple phases of the cardiac cycle, cardiac micro-CT can produce 4D images of the beating heart. These datasets can be used to measure cardiac function, including ventricular volumes, stroke volume, ejection fraction, wall motion, and cardiac output (Wetzel et al. 2007, Badea et al. 2005, 2007, 2008c, 2011b). Measurements of cardiac function by micro-CT can be used, for example, to evaluate the effect of drugs such as dobutamine-induced cardiac stress (see Figure 36.8a (Badea 2011b)). Cardiac micro-CT can also be used to longitudinally measure changes in cardiac function over time. For example, left ventricular remodeling following a coronary ligation-induced myocardial infarction has been tracked by micro-CT (Sheikh et al. 2010). Measurements of cardiac function and infarct size have also been performed in coronary ligation mouse models using either a combination of blood pool agent and a low molecular contrast agent (Nahrendorf et al. 2007) or a blood pool contrast agent (Exia 160), which shows specific uptake in myocardium (Ashton et al. 2014a). An example of micro-CT imaging of myocardial infarction using a delayed hyper-enhancement protocol in a rat, as described in Nahrendorf et al. 2007, is shown in Figure 36.8b.

\subsubsection{Liver Imaging}

Blood pool contrast agents, which avoid renal clearance due to their large size $(>6 \mathrm{~nm})$, are eventually cleared from the bloodstream by phagocytic cells in the reticuloendothelial system (Moghimi et al. 2001). This clearance occurs primarily in the liver and spleen, which leads to accumulation of contrast in those organs over time. This provides high enhancement of these organs for liver and spleen-specific imaging. One of the commercially available micro-CT contrast agents, Fenestra LC (http:// www.medilumine.com/), is composed of iodinated phospholipids, which are recognized by the ApoE receptor on hepatocytes and internalized in the liver, which provides additional specificity for liver imaging. Because these blood pool contrast agents are taken up by normal-functioning liver and spleen, they can be used to identify necrotic regions (Chouker et al. 2008), liver tumors (Almajdub et al. 2007, Montet et al. 2007, Desnoyers et al. 2008, Graham et al. 2008, Kim et al. 2008, Boll et al. 2011b), and spleen tumors (Almajdub et al. 2007), as well as to measure organ volume, quantify hepatic necrosis (Varenika et al. 2013), and determine liver anatomy (Fiebig et al. 2012). Figure 36.9
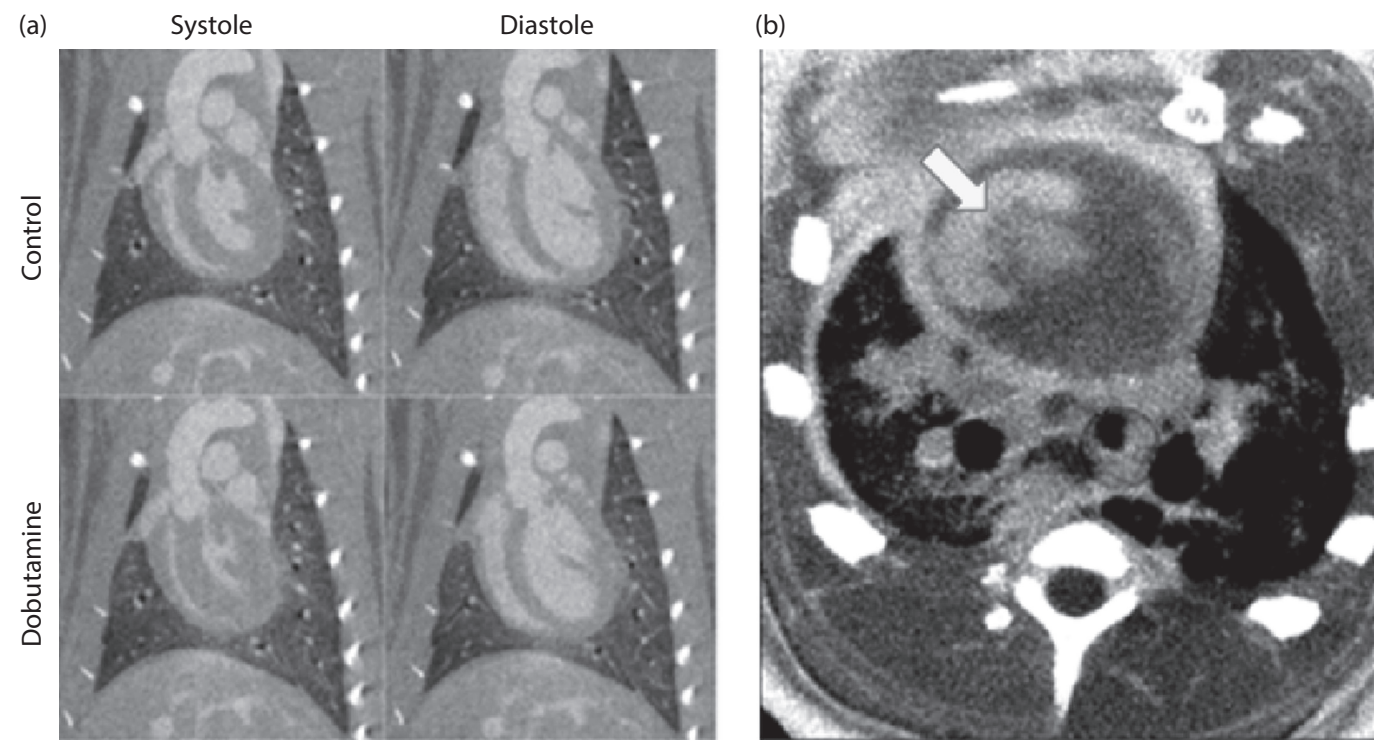

FIGURE 36.8 (a) Coronal micro-computed tomography (micro-CT) images through the left ventricle showing the heart in systole (left images) and diastole (right images) with and without the administration of dobutamine $(10 \mu \mathrm{g} / \mathrm{kg} / \mathrm{min})$. End systolic volume is significantly decreased, and stroke volume and cardiac output are both significantly increased. End diastolic volume is relatively unchanged after administration of dobutamine. (b) An axial image showing myocardial infarction (arrow) in a rat using delayed hyper-enhancement. 
(a)

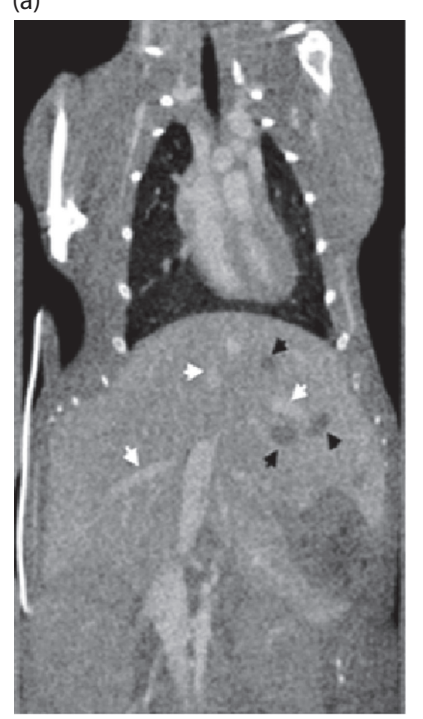

(b)

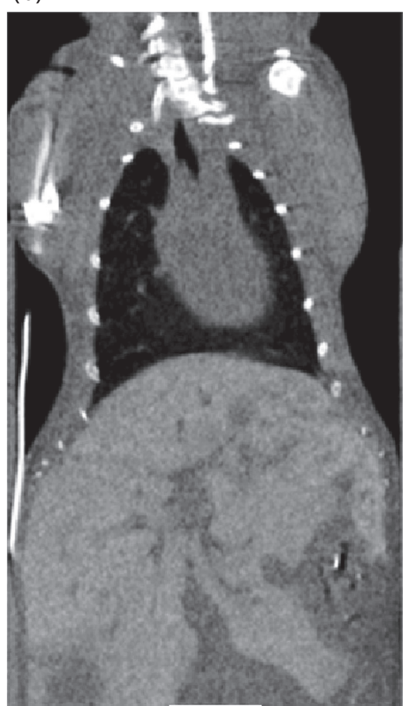

FIGURE 36.9 Contrast-enhanced micro-computed tomography (microCT) images of mice with HT29 human colon adenocarcinoma acquired (a) day 1, (b) day 5 post-contrast injection. Arrowheads in (a) indicate tumors (black) and blood vessels (white). A liposomal iodinated contrast agent has been used. (Reproduced from Pandit, P. et al. 2013. Acad Radiol 20(4), 430-439.)

shows slices in a coronal orientation of a liver in mice with HT-29 colon adenocarcinoma tumors (Pandit et al. 2013). The mouse was injected with a liposomal iodinated contrast agent and imaged one day and five days post-injection. The tumors appear hypo-enhanced relative to normal liver parenchyma.

\subsubsection{Kidney Imaging}

Micro-CT imaging of the kidneys can provide important anatomical and even functional data. Because any low molecular weight iodinated contrast agents undergo renal excretion, contrast enhancement of the kidneys is straightforward. Thus, abnormalities like renal cysts are readily detected and measured during longitudinal studies (Xu et al. 2013). Research on mouse models for renal diseases, such as polycystic kidney disease, also use micro-CT (Wilson 2008).

Micro-CT allows accurate measurements of kidney volume $\left(\mathrm{mm}^{3}\right)$, length $(\mathrm{mm})$, and thickness (mm) (Almajdub et al. 2008). Figure 36.10 presents a maximum intensity projection of both kidneys and a coronal micro-CT slice through one of the kidneys. Note that the renal pelvis (inner core structure) is immediately distinguishable from the renal cortex (outer rim) and medulla (intermediate space in between).

\subsubsection{Gastrointestinal Imaging}

Micro-CT has demonstrated its usefulness in detecting colon lesions in mouse models of cancer. Negative contrast-enhanced micro-CT imaging of murine models of colonic tumors has proven to be useful for detecting lesions $<2 \mathrm{~mm}$, validating the modality as a longitudinal imaging platform for investigating the disease (Pickhardt et al. 2005, Durkee et al. 2008). Other studies have investigated the efficacy of multiple contrast-enhanced experiments for (a)

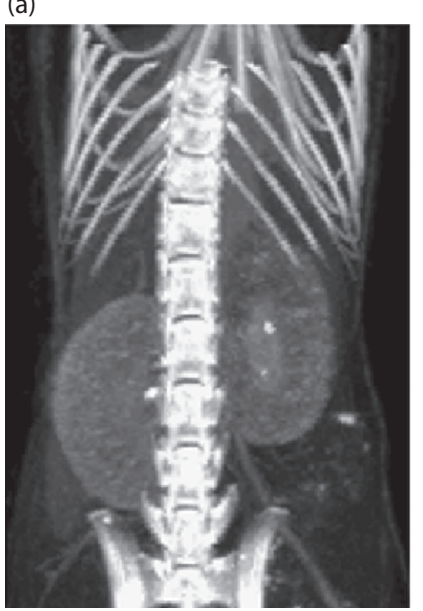

(b)

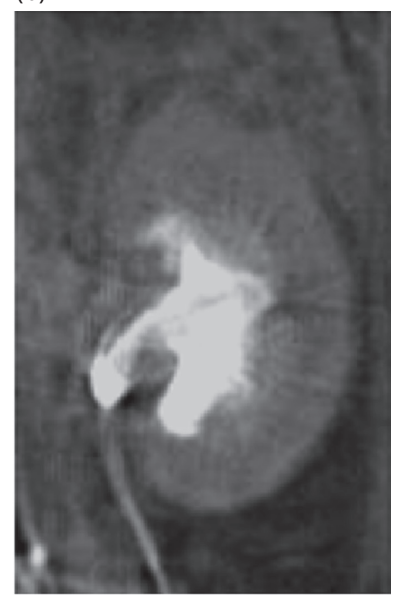

FIGURE 36.10 Micro-computed tomography (micro-CT) images of murine kidneys with low molecular weight contrast enhancement. (a) A maximum intensity projection and (b) a coronal section of a single kidney. The renal cortex is noted along the outer rim of the kidneys due to its different uptake than the adjacent medulla.

detecting colon malformations. A combination of intraperitoneal (IP) injected with rectally-administered iodinated contrast agents was used for detecting colon tumors (Choquet et al. 2007).

Barium sulfate $\left(\mathrm{BaSO}_{4}\right)$ solution is a contrast agent used for imaging the gastrointestinal (GI) tract. In clinical use, $\mathrm{BaSO}_{4}$ is suspended and then ingested orally for upper-GI imaging, or a $\mathrm{BaSO}_{4}$ solution is administered rectally for lower-GI imaging. $\mathrm{BaSO}_{4}$ coats the lining of the esophagus, stomach, and intestines for a length of time sufficient for CT imaging. Figure 36.11 presents examples of GI imaging using micro-CT with $\mathrm{BaSO}_{4}$. (Wathen et al. 2013). Polyps in the colon can also be detected by using an administration of $\mathrm{BaSO}_{4}$ followed by air used as a negative contrast agent for the bowel space (Boll et al. 2011a).

\subsubsection{Cancer Imaging}

Because tumors generally have the same density as their surrounding tissues, contrast agents are necessary for tumor (a)

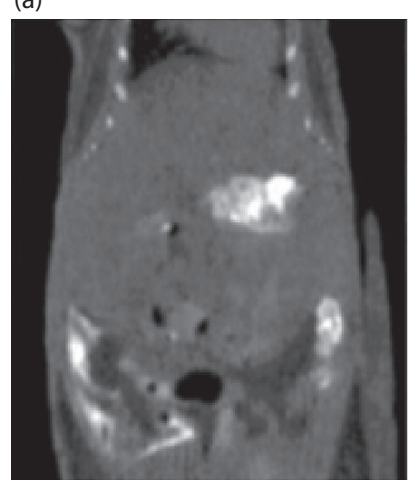

(b)

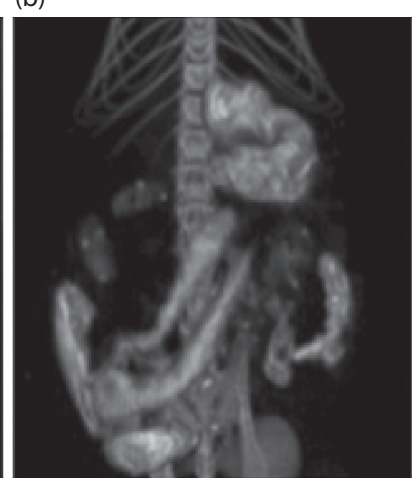

FIGURE 36.11 Micro-computed tomography (micro-CT) data of a murine gastrointestinal (GI) tract with $\mathrm{BaSO}_{4}$. In the coronal image (a), loops of the small intestine can be seen. (b) 3D reconstruction colored to aid in the visualization of the gastrointestinal (GI) tract (Reproduced from Wathen, C. A. et al. 2013. Sensors (Basel) 13(6), 6957-6980.) 
identification and characterization by micro-CT. The vast majority of cancer imaging studies have been performed using blood pool nanoparticle contrast agents. Nanoparticles tend to accumulate in tumors due to the enhanced permeability and retention (EPR) effect (Maeda 2001, Maeda et al. 2000). Rapid angiogenesis within a tumor leads to the development of immature, poorly organized, leaky vasculature. Gaps in this leaky vasculature are large enough that nanoparticles (up to $300 \mathrm{~nm}$ ) can extravasate into the tumor tissue. Tumors also tend to have very poorly developed lymphatic drainage, so the nanoparticles are not cleared from the tumor once they extravasate. This effect leads to the gradual passive accumulation of nanoparticles in the tumor perivascular space over the course of hours to days. EPR has been widely exploited for both tumor imaging and therapy using nanoparticle agents.

Using micro-CT, dynamic biodistribution of contrast agent within small animal tumor models can be tracked. A liposomal iodine contrast agent was used in a rabbit tumor model for contrast agent tracking and biodistribution analysis (Zheng et al. 2009). Quantitative analysis was performed to determine the percent contrast agent uptake within each organ, including the tumor. Liposomal iodine was also used in two mouse models of breast cancer to demonstrate dynamic changes in enhancement within tumor vasculature and tumor parenchyma (Samei et al. 2009, Ghaghada et al. 2011). Immediately after injection, the contrast agent is entirely contained within the vasculature, with no significant enhancement within the tumor tissue. This early phase allows for the analysis of tumor vascular morphology, location, and density. After the contrast agent is cleared from the bloodstream, late-phase imaging can be performed to demonstrate the passive accumulation of the contrast agent in the tumors due to EPR. The tumors typically show heterogeneous enhancement throughout their volumes, demonstrating spatial heterogeneity in tumor perfusion and vascular permeability. A few studies have been done in mouse xenograft tumor models to carefully map the spatial and temporal distribution of liposome uptake by micro-CT (Ekdawi et al. 2015), which has important implications for nanoparticle-based drug delivery. Figure 36.12 shows an example of early- and delayedphase imaging using liposomal iodine-based contrast agent (Ghaghada et al. 2011). Measurements of tumor vascular density in early-phase imaging and total contrast accumulation in late-phase imaging have also been used in two mouse models of lung cancer to differentiate different aggressiveness levels in tumors (Badea et al. 2012). Iodine-containing nanoparticle contrast agents have also been used for tumor imaging in two other models of lung cancer (Kindlmann et al. 2005, Anayama et al. 2013) and a mouse model of liver cancer (Rothe et al. 2015). Gold nanoparticles have also been used for passive tumor targeting in mouse models of breast and brain cancer (Hainfeld et al. 2006, 2013).

\subsubsection{Micro-CT with Active Targeting}

Beyond the passive targeting of nanoparticles to the reticuloendothelial system or tumors, active targeting of nanoparticles can be accomplished by conjugating specific ligands to the nanoparticle surface which can then link to their binding partners in vivo (Erathodiyil and Ying 2011). Typically, these (a)

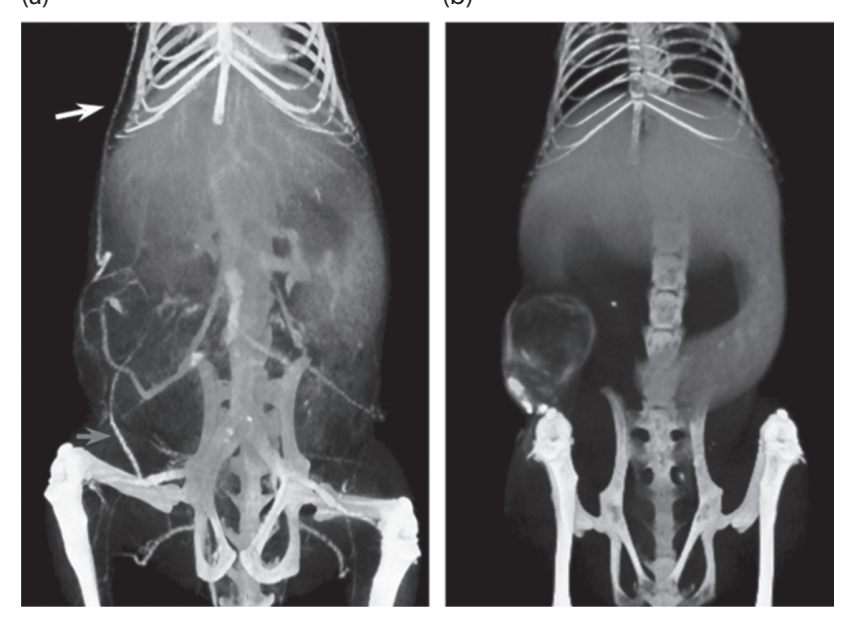

FIGURE 36.12 (a) Coronal 3D volume-rendered images demonstrating co-opted vessels (top arrows) and newly developed tumor vessels (bottom) in early phase imaging (immediately after nanoparticle contrast agent administration). Both the co-opted and newly developed vessels traced their origin back to major venous structures such as the jugular vein (origin of vessel labeled with top arrow) or the inferior vena cava (origin of vessel labeled with bottom arrow). (b) In the delayed micro-CT image, at five days postliposomal contrast injection, the tumor is clearly enhanced. The only other organs enhanced are the liver and spleen, which are the organs for nanoparticle contrast agent clearance.

binding partners are cellular receptors or extracellular matrix proteins that are overexpressed in a pathological condition, so binding is specific to the region of pathology. Potential ligands for conjugation to the nanoparticle surface include antibodies, antibody fragments, other proteins, peptides, aptamers, lipids, carbohydrates, and other small molecules. The use of targeted contrast agents for micro-CT has recently been reviewed ( $\mathrm{Li}$ et al. 2014). Gold nanoparticles have been used extensively for active targeting due to the ease of gold surface modification via gold-thiol bond formation. Gold nanoparticles have been used as a micro-CT contrast agent for the targeting of multiple tumor markers, including Her2 (Hainfeld et al. 2011), the gastrin-releasing peptide (GRP) receptor (Chanda et al. 2010), the epidermal growth factor receptor (EGFR) (Reuveni et al. 2011), the folic acid receptor (FAR) (Wang et al. 2013), and tumor microcalcifications (Cole et al. 2014). Figure 36.13 demonstrates the use of EGFR-antibody conjugated gold nanoparticles to target an EGFR-expressing subcutaneous tumor. Tumor enhancement was significantly increased with targeted gold nanoparticles compared to non-targeted gold nanoparticles (190 HU vs. $78 \mathrm{HU}$ ).

Gold nanoparticles have also been used for CT imaging of lymph nodes by targeting CD4 (Eck et al. 2010), imaging of inflammation by targeting intravascular E-selectin (Wyss et al. 2009), imaging of atherosclerosis by targeting fibrin (Winter et al. 2005), imaging of myocardial scars by targeting collagen (Danila et al. 2013), and imaging of other cardiovascular disease (Ghann et al. 2012). In addition to targeting by the surface conjugation of a ligand, some nanoparticles have inherent targeting abilities due to their nanoparticle chemistry. Gold nanoparticles encapsulated within high-density lipoprotein (HDL) particles are naturally recognized by HDL 
(a)

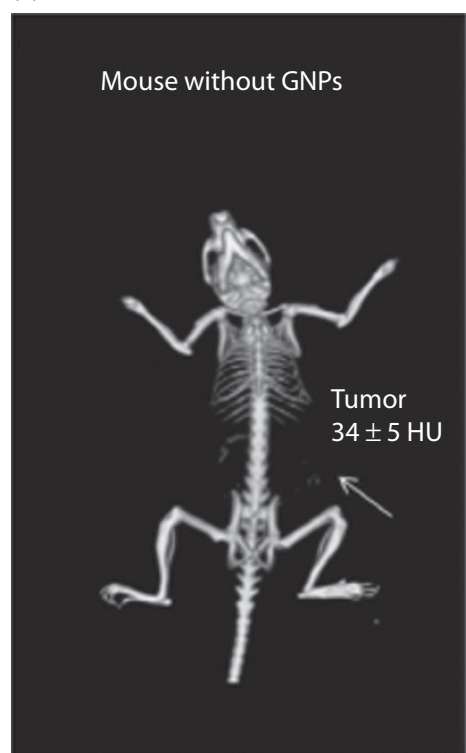

(b)

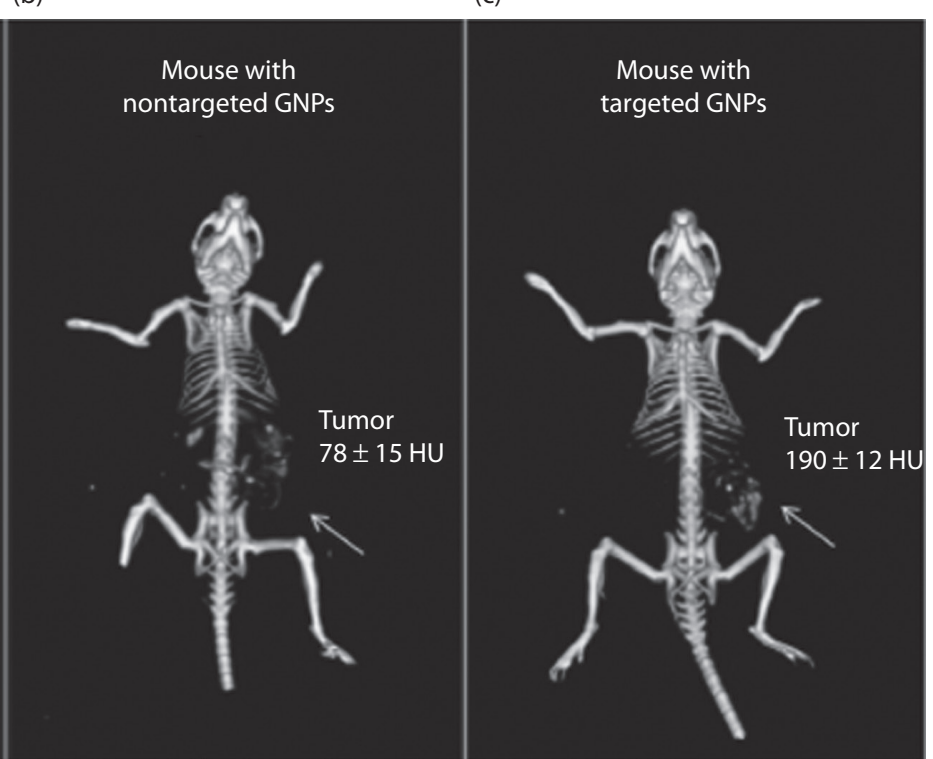

FIGURE 36.13 3D reconstructions of micro-computed tomography (micro-CT) images of a mouse subcutaneous tumor model. Mice were injected with saline (a), non-targeted gold nanoparticles (b), or epidermal growth factor receptor (EGFR)-antibody targeted gold nanoparticles (c). Increased CT enhancement was seen for both types of nanoparticles, but targeted nanoparticles showed significantly higher enhancement than non-targeted controls. (Reprinted from Reuveni, T. et al. 2011. Int J Nanomedicine 6, 2859-2864.)

receptors and taken up in atherosclerotic plaques (Cormode et al. 2010). Exia-160 consists of iodinated molecules that can be fully metabolized by the body, and therefore it accumulates in metabolically active tissues, including the myocardium and brown adipose tissue. This effect has been used to discriminate between healthy and infarcted myocardium (Ashton et al. 2014a).

\subsection{Multi-Modality Imaging}

Micro-CT can be used together with other imaging modalities in order to better study functional molecular and anatomical information simultaneously. A micro-CT system can be combined with single photon emission computed tomography (SPECT), positron emission tomography (PET), or fluorescence molecular tomography (FMT) into a single unit (Goertzen et al. 2002, Liang et al. 2007). SPECT, PET, and FMT are all highly sensitive, so targeted molecular imaging with radio-labeled or fluorescently-labeled small molecules or biomolecules is readily accomplished. However, these modalities are all limited by poor spatial resolution and poor anatomical imaging. By combining these systems with micro-CT, high-resolution anatomical images can be co-registered with molecular images to produce highly useful datasets. Combining micro-SPECT and micro-PET with micro-CT can also improve the image quality of the resultant SPECT and PET images by allowing for attenuation correction (Chow et al. 2005, Hwang and Hasegawa 2005). Figure 36.14 shows a combined micro-CT/micro-PET image for a 4T1 breast tumor-bearing mouse after injections of both liposomal iodine and ${ }^{18} \mathrm{~F}$-fluorodeoxyglucose. The multi-modal imaging took place 72 hours post-injection of the liposomal CT contrast agent. Note the co-localization of the EPR-based accumulation of the iodinated liposomes visualized by micro-CT and the FDG uptake in the micro-PET image.

A second application of multi-modal imaging, which has gained much attention recently, is the use of agents that produce contrast for multiple imaging modalities simultaneously. Thus, multiple imaging modalities can be used after injection of a single contrast agent. This helps to improve registration between the different modalities and increase the amount of information gained from hybrid imaging systems. Many different formulations of multi-modal contrast agents have been developed, and the development of these agents has been reviewed previously (Key and Leary 2014). Combined CT/MR contrast agents have been developed using gadolinium chelates conjugated to gold nanoparticles (Alric et al. 2008) or gold nanoshells (Coughlin et al. 2014), liposomes containing both gadolinium and iodinebased contrast agents (Zheng et al. 2006), and iron oxide core nanoparticles surrounded by either a gold shell (Carril et al. 2014) or a mesoporous silica shell filled with iodinated oil (Xue et al. 2014). A combined CT/SPECT agent has been developed using a dendrimer linked to both iodinated organic molecules and SPECT agent chelators (Criscione et al. 2011). A combined PET/CT agent has been demonstrated using gold nanoparticles conjugated to both glucose and ${ }^{18} \mathrm{~F}$-fluorodeoxyglucose (FDG) for targeting of metabolically active tumors (Roa et al. 2012, Feng et al. 2014). All of these formulations have been successfully tested in vivo with multi-modal small animal imaging.

\subsection{Theranostics}

Many nanoparticles used as micro-CT contrast agents can easily be adapted to provide, or incorporate, therapeutics thus serving in theranostics. Gold nanoparticles, for example, have the 
(a)

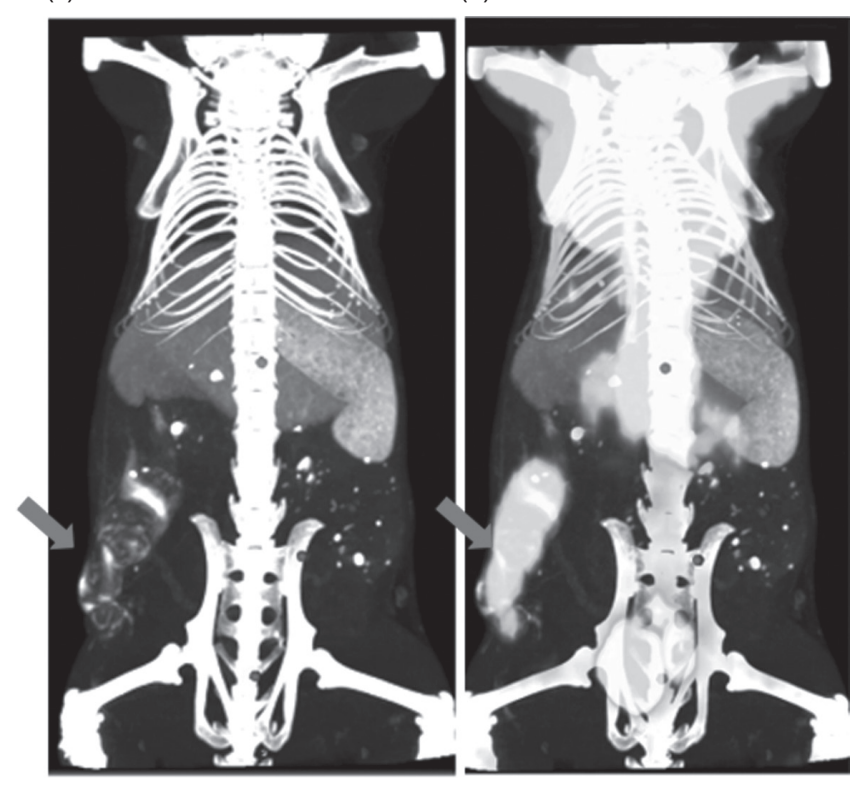

FIGURE 36.14 An illustration of a multi-modality study using micropositron emission tomography-micro-computed tomography (micro-PETmicro-CT) in a mouse with 4T1 breast tumors (see arrows). (a) The maximum intensity projection (MIP) of the delayed enhanced micro-CT image at 72 hours post-injection of a liposomal iodinated contrast agent. (b) The overlapping of MIPs for micro-CT and the ${ }^{18} \mathrm{~F}$-fluorodeoxyglucose (FDG)micro-PET. Note that there is a significant accumulation of the iodinated liposomes as a result of enhanced permeability and retention in the tumor. The micro-CT image provides high-resolution anatomical detail to give context to the tumor signal seen in the micro-PET image (Reproduced from Badea, C. T. et al. 2011a. Multi-modality PET-CT imaging of breast cancer in an animal model using nanoparticle X-ray contrast agent and 18F-FDG. Medical Imaging 2011: Biomedical Applications in Molecular, Structural, and Functional Imaging 7965.)

inherent ability to increase the effectiveness of radiation therapy because they absorb therapeutic X-rays efficiently and then release that energy to the surrounding tissues, thus increasing the locally delivered dose in regions of high nanoparticle concentration. This radiation augmentation has been studied by several groups to effectively treat cancer in multiple animal models (Hainfeld et al. 2004, 2008, 2010, 2013, 2014, Jeremic et al. 2013, Park et al. 2015, Wolfe et al. 2015). Gold nanoparticles also exhibit high absorbance of light at their surface plasmon resonance wavelength, which can be tuned by altering the shape and size of the nanoparticle. For many gold nanoparticle shapes (i.e., nanorods, nanoshells, nanostars), this plasmon resonance occurs in the near infrared region, which is optimal for use with photothermal heating. In photothermal heating, nanoparticles convert laser light into heat, which leads to local hyperthermia. This effect can be used for tumor ablation. The use of nanoparticles for combined CT imaging and photothermal therapy has been recently reviewed (Curry et al. 2014). Gold nanorods (Huang et al. 2011) and hollow gold nanoshells (Park et al. 2015) have both been used for combined CT imaging, radiation therapy, and photothermal therapy. Figure 36.15 shows a gold nanostar theranostic probe that was used for CT imaging and photothermal therapy in a mouse model of primary soft tissue sarcoma (Liu et al. 2015). This probe showed high tumor accumulation and CT

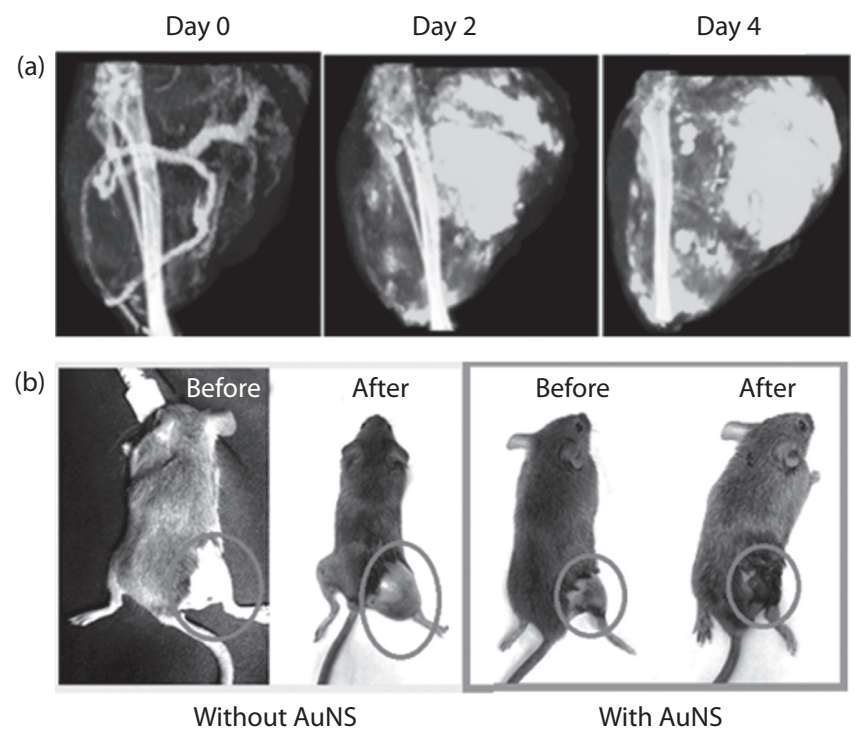

FIGURE 36.15 (a) Micro-computed tomography (CT) maximum intensity projections through the soft-tissue sarcoma on a mouse hindlimb at Days 0,2 , and 4 post-injection of gold nanostars (AuNS). Green represents gold concentration (windowed from $2 \mathrm{mg} / \mathrm{mL}$ to $10 \mathrm{mg} / \mathrm{mL}$ ). (b) Photothermal therapy after injection of either gold nanostars or saline. The mice receiving gold nanostars showed complete remission of their sarcoma, while the control mice had continued rapid tumor growth.

enhancement, as well as effective tumor ablation following photothermal therapy. Therapeutics can also be incorporated into nanoparticles by direct conjugation to the nanoparticle surface or by co-encapsulation of the therapeutic with the imaging agent (e.g., within the aqueous core of a liposome). Both methods have been used for the addition of therapeutic radioisotopes or chemotherapeutics to nanoparticle contrast agents (Chen et al. 2014, Lu 2014, Ryu et al. 2014, Zhu et al. 2014).

\subsection{Spectral Micro-CT}

Spectral information can improve contrast discrimination in CT imaging. In traditional CT imaging, the overall attenuation of X-ray intensity is measured by the detector, but the detected $\mathrm{X}$-rays are not spectrally resolved. The spectrum of transmitted $\mathrm{X}$-rays is important because the absorption of X-rays by different materials is highly dependent on X-ray energy, so the transmitted X-ray spectrum depends on what materials are present along the X-ray path. Therefore, there is a significant amount of information that can be gained by including spectral data in the CT reconstruction process. Based on differences in X-ray absorption, multiple materials can be differentiated and quantified within a single scan using spectral CT.

There are two primary methods used to obtain spectral CT data. The first method, dual energy (DE) CT, uses X-ray sources with two different energy spectra and traditional energy integrating X-ray detectors. The second method uses a single X-ray source, but has energy-resolving detectors (photon-counting detectors) that can measure the energy of each detected photon. Dual energy CT is currently used clinically and has been successful in improving imaging for a variety of applications (Jepperson 


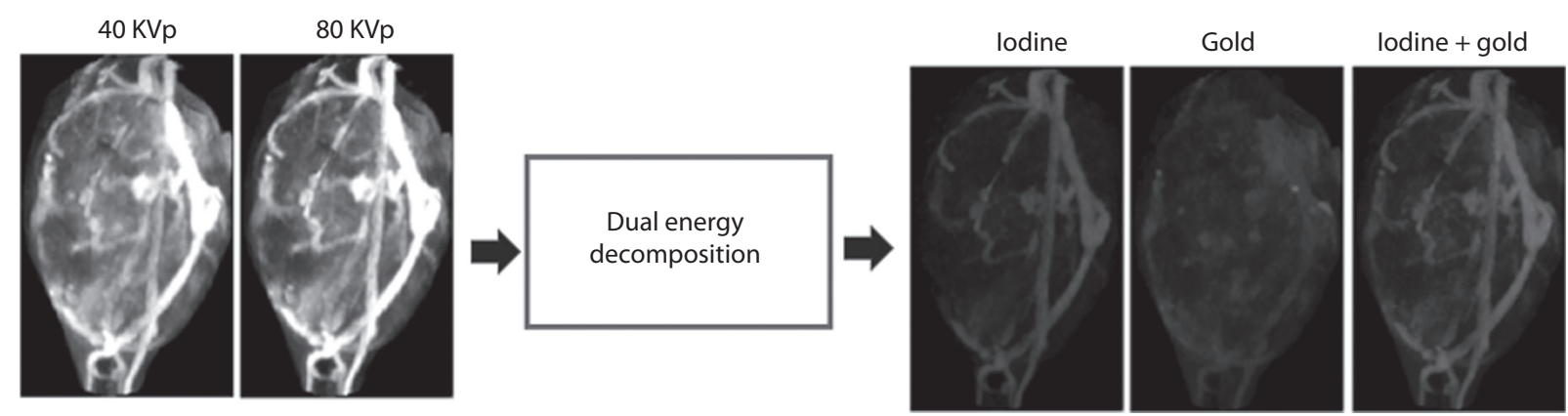

FIGURE 36.16 Dual energy micro-computed tomography (CT) scans and material decompositions for an in vivo imaging of gold nanoparticles and iodine-containing liposomes within a mouse soft-tissue sarcoma. The iodine (shown in red) and gold (shown in green) maps are the result of dual energy decomposition. The decomposition is able to successfully differentiate signal from the gold and iodine contrast agents.

et al. 2013, Aran et al. 2014, Bongartz et al. 2014, Marin et al. 2014, Mileto et al. 2014, Ohana et al. 2014, Paul et al. 2014).

DE CT can use either a single X-ray source, which rapidly switches between two tube voltages, or two separate sources/ detectors which are orthogonally positioned and each operate at a different voltage. In either case, X-ray projections are acquired at each rotation angle using both X-ray sources. Additionally, a sandwich detector is sometimes used to separate low-energy and high-energy X-rays. In DE CT, two complete CT datasets are acquired for two different X-ray energy spectra. Most of a subject's body appears the same on both images, because absorption of X-rays by low atomic weight materials, which is primarily due to Compton scattering, is very weakly dependent on X-ray energy. However, the photoelectric effect in high atomic weight materials is highly dependent on X-ray energy. Therefore, the attenuation coefficient of high atomic weight materials (calcium in bone, iodine, gold) will depend on the energy spectrum of the incident X-rays. This effect is particularly pronounced if the two energy spectra fall on either side of the k-edge for one of the materials. Because there is a large increase in attenuation at energies above the k-edge, this leads to a large difference in signal between the two scans. By combining data from the two energy sets, these high $\mathrm{Z}$ materials can be differentiated from one another and quantified.

Spectral separation using DE CT is somewhat limited by our ability to minimize the overlap of X-ray spectra using polychromatic sources. The separation between the two energy spectra can be improved by applying additional filtration to the X-ray tubes, which can preferentially remove low-energy photons and further increase the average energy of the X-ray spectrum. The other limitation for DE CT is its ability to discriminate between closely-related elements, for example, barium and iodine.

Using this method, DE micro-CT has been used successfully for a variety of applications in mice. DE micro-CT was used for atherosclerosis imaging to differentiate liposomal iodine accumulated in plaque macrophages from calcium within the plaque (Bhavane et al. 2013). Iodine accumulated within the myocardium has been separated from other soft tissues and from calcium in the bone for imaging of myocardial infarction (Ashton et al. 2014a). DE micro-CT has been used to separate gold nanoparticles accumulated within soft-tissue sarcomas (Clark et al. 2013) or primary lung tumors (Ashton et al. 2014b) from liposomal iodine within the vasculature. In these studies, the simultaneous measurement of two different nanoparticle concentrations was used to calculate tumor vascular density and vascular permeability. This process is demonstrated in Figure 36.16, which shows scans of sarcoma tumors in a mouse that was injected first with gold nanoparticles and 72 hours later with iodinated liposomes. The dual energy micro-CT was performed using 40 and $80 \mathrm{kVp}$. Following the acquisition, two 3D images were reconstructed. These two scans were then mathematically decomposed into maps of iodine and gold concentrations (Clark et al. 2013).

Validation of DE micro-CT decompositions was performed using histology and ex vivo measurements of tissue gold and iodine concentrations (Ashton et al. 2014b). In two additional studies, DE micro-CT was used to assess vascular changes following radiation therapy. In the first, the increase in vascular permeability in a soft-tissue sarcoma was determined by measuring accumulation of liposomal iodine (Moding et al. 2013). In the second study, cardiac injury following radiation therapy was assessed using gold nanoparticles and liposomal iodine (Lee et al. 2014). Our Duke group has recently also demonstrated triple-energy micro-CT for the differentiation of three materials: gold, iodine, and gadolinium. Using a novel algorithm called spectral diffusion (Clark and Badea 2014), these three materials were successfully separated and quantified both in an in vitro phantom and in vivo.

Although PCXDs are still experimental for clinical CT, their use in preclinical small animal studies has been successfully demonstrated. Spectral CT has been used with targeted nanoparticles to image atherosclerotic plaques (Cormode et al. 2010). Gold nanoparticles were encapsulated within HDL particles to target plaque macrophages. A preclinical spectral CT system (Phillips Research, Hamburg) was used to differentiate the gold from iodine, calcium, and soft tissues. Spectral micro-CT was used to resolve the signals from gold, iodine, and calcium within the tissue phantom matrix. The spectral micro-CT system successfully differentiated the phantom regions containing gold, iodine, and calcium, with very little overlap between the signals. They also tested the targeting of their gold-HDL particles in a mouse model of atherosclerosis. Spectral micro-CT (and subsequent histology) demonstrated that the gold successfully accumulated within the plaques and that gold could be discriminated from iodine, calcium, and soft-tissue in vivo. The same HDLencapsulated gold nanoparticles have been used along with a blood pool iodine contrast agent to simultaneously image the signals from gold accumulated within lymph nodes, and iodine within the blood, bone, and soft tissue (Roessl et al. 2011). Gold 
nanoparticles accumulating in lymph nodes after subcutaneous injection have been differentiated from soft tissue and bone (Schirra et al. 2012). Iodine within the vasculature and barium within the GI tract have been imaged and differentiated from bone and soft tissue (Anderson et al. 2010). Spectral imaging has also been used to detect novel ytterbium nanoparticles within the vasculature (Pan et al. 2012) and organic bismuth nanocolloids targeted to fibrin-rich clots (Pan et al. 2010). In both cases, spectral CT was used to differentiate contrast agent signal from soft tissue and bone. The primary limitation in all of these studies was that the low photon-count rate limitations of the PCXD system resulted in a long scan time. Because the scan time was so long, the imaging was done after sacrificing the animals in order to prevent motion over the course of the long acquisition. Despite the limitations, these studies demonstrate that spectral CT using a PCXD system has the potential for high-quality in vivo imaging and material discrimination. Some technical problems remain to be solved, but PCXD systems have great promise for use in both preclinical and clinical CT imaging.

\subsection{Phase Contrast Micro-CT Imaging}

Micro-CT creates the perfect environment for the development of phase contrast CT imaging. For most micro-CT applications, $\mathrm{X}$-ray photons are treated as particles. Treating X-rays instead as electromagnetic waves, their complex index of refraction is expressed as $n=1-\delta-i \beta$, which relates to the linear absorption coefficient, $\mu_{0}$, as $\beta=\mu_{0} *(\lambda / 4 \pi)$ and to the coefficient of phase change, $\eta$, as $\delta=\eta^{*}(\lambda / 2 \pi)$ for X-ray wavelength, $\lambda$. For diagnostic X-rays (10-100 keV) and low atomic number elements $(\mathrm{Z} \leq 20), \delta$ is approximately two to three orders of magnitude larger than $\beta$ and falls off more slowly with increasing X-ray energy $\left(\sim 1 / \mathrm{E}^{2}\right.$ vs. $\left.\sim 1 / \mathrm{E}^{3}\right)$. Because of these factors, phase contrast is interesting for imaging soft biological tissues without the need for exogenous contrast agents and with potentially reduced radiation dose (Momose 2005, Bravin et al. 2013).

These theoretical advantages have been practically validated using monochromatic, synchrotron X-ray sources and one of several imaging schemes, including (1) propagation-based, (2) analyzer-crystal-based, and (3) grating interferometer-based phase contrast. Propagation-based phase contrast imaging relies on the propagation distance of X-rays after they have been refracted within the sample (i.e., on the object-to-detector distance). Rotating the sample and acquiring projections at a single distance allows reconstruction of hybrid, edge-enhanced data in which the absorption information is augmented by the Laplacian of the refractive index. Explicit phase and absorption retrieval are possible by acquiring the projections at several object-to-detector distances followed by algorithmic post-processing (Langer et al. 2008). Analyzer-crystal-based methods rely on Bragg diffraction within a silicon single crystal. Acquiring projections with a fixed sample position as the angle of the analyzer crystal is varied with respect to the X-ray beam exiting the sample yields a rocking curve (crystal angle vs. recorded intensity) from which refraction and apparent absorption projections can be recovered (Chapman et al. 1997). Grating interferometer-based phase contrast methods use aligned, micron-scale gratings to generate systematic interference fringes in the propagating X-ray wavefront. Refraction within the sample shifts the interference fringes, allowing recovery of the differential of phase shifts caused by the object from intensity curves generated by stepping the sample or a component grating through a grating period (phase stepping), while the sample position remains fixed (David et al. 2007).

One of the primary challenges in translating these phase contrast imaging methods from synchrotron beam lines to routine clinical and preclinical application is the comparatively low brilliance and low coherence of laboratory X-ray sources; however, technological developments is this area are very promising. Pfeiffer et al. have demonstrated the effective translation of the Talbot-Lau grating interferometer from a synchrotron source to a polychromatic X-ray tube source through the addition of a source grating, which collimates the source beam into an array of individually coherent X-ray line sources (Pfeiffer et al. 2006). They have also demonstrated the recovery of three contrast mechanisms with a single scan (absorption contrast, differential phase contrast, and dark field scatter contrast) (Pauwels et al. 2012, Bech et al. 2013) and have developed a prototype small animal scanner in collaboration with Bruker micro-CT (Tapfer et al. 2012, Pauwels et al. 2012). Additionally, they have demonstrated the potential value of differential phase contrast and dark field imaging in several applications, including cancer identification and classification (Tapfer et al. 2013, Willner et al. 2014) and lung imaging (Meinel et al. 2014).

As an alternative to the Talbot-Lau interferometer with source grating, a non-interferometric, grating-based approach for differential phase contrast with a laboratory source, called coded aperture X-ray phase contrast imaging, has been proposed and demonstrated using a physical phantom (Munro et al. 2012). Additional work has been done with an analyzer crystal-based setup and a laboratory source (Connor et al. 2011).

Several technical challenges must be overcome prior to the widespread adoption of phase contrast imaging. High-quality tomographic reconstructions of differential phase contrast data using a Talbot-Lau grating interferometer can require hours to tens of hours of scanning owing to the flux limitations associated with laboratory X-ray sources, which are exacerbated by collimator-like phase gratings, and owing to the phase-stepping performed at each sample rotation angle (Fu et al. 2014). A single-shot alternative to phase-stepping, which uses Moiré patterns and Fourier analysis, has been demonstrated using a physical phantom and could lead to significantly reduced scan times (Bevins et al. 2011). Phase wrapping in thick specimens and bone poses a significant hurdle for preclinical and clinical imaging; however, algorithms have already been proposed and demonstrated for phase unwrapping (Tapfer et al. 2012, Epple et al. 2013). Additional fabrication challenges are associated with scaling the field-of-view and viable energy range for grating interferometers. Proposed solutions to these problems include helical scanning (Fu et al. 2014) and inclining the gratings with respect to the beam direction for reduced transmittance of high-energy photons (Stutman et al. 2013), respectively.

In addition to technological developments, the adaptation of iterative reconstruction algorithms to the problem of tomographic phase contrast image reconstruction will reduce associated scanning times and radiation dose. Some work has been presented in the literature illustrating how existing iterative CT reconstruction algorithms can be modified for this purpose (Köhler et al. 2011, 


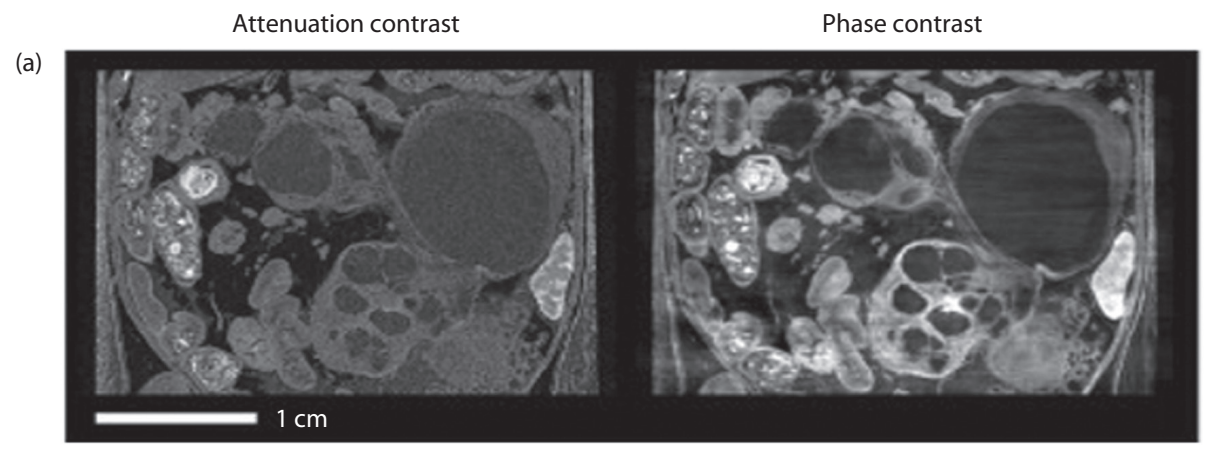

(b)

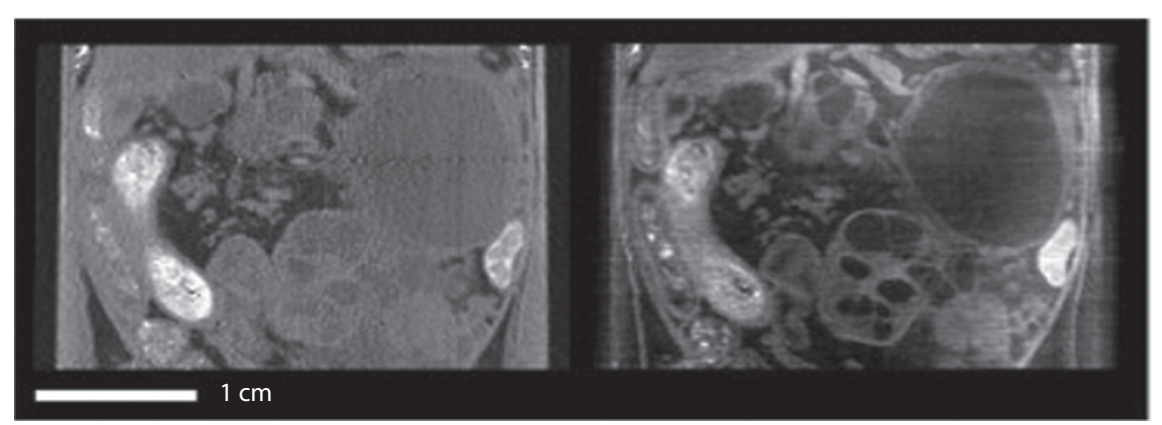

FIGURE 36.17 Coronal micro-computed tomography (CT) slices corresponding to attenuation and phase contrast of the abdominal area in the mouse using (a) synchrotron, (b) tube source. All results shown in this Figure 36.17 were acquired ex vivo after whole-animal perfusion fixation with paraformaldehyde. (Reproduced from Tapfer, A. et al. 2013. PloS ONE 8(3), e58439. With permission.)

Fu et al. 2013). Furthermore, techniques for enforcing gradient sparsity in phase contrast data have been demonstrated (Nilchian and Unser 2012, Herzen et al. 2014).

Figure 36.17 is adapted from Tapfer et al. 2013 and compares phase and absorption contrast results using a synchrotron and laboratory source in a mouse model of pancreatic ductal adenocarcinoma. The authors have used a grating-based phase contrast CT method. It was found both visually and quantitatively by means of contrast to noise ratio that the increased soft tissue contrast apparent in phase images does allow for tumor identification, unlike in the attenuation images. Note that the quality of images acquired with a conventional X-ray tube is not much inferior to the synchrotron acquisition.

\subsection{Radiation Dose}

One of the major limitations of X-ray CT imaging is exposure to radiation. High radiation doses are required for high-resolution CT scans. Signal-to-noise ratio in CT is inversely proportional to the square root of the number of X-rays passing through each voxel. As voxel size decreases, the number of X-rays necessary to maintain a constant signal-to-noise ratio increases significantly. In planning micro-CT studies, a balance must be made between desired image quality and radiation exposure.

$\mathrm{X}$-ray radiation exposure can lead to biological damage and long-term health effects (Boone et al. 2004). The $\mathrm{LD}_{50 / 30}$ radiation dose in mice (the dose required to kill $50 \%$ of mice within 30 days) depends on many factors, but tends to be between 5 and 8 Gy (Ritman 2004, Carlson et al. 2007). The typical radiation dose for a single micro-CT scan can vary widely and reported values in the literature range from $0.017-0.78 \mathrm{~Gy}$ (Carlson et al.
2007). Rodents have the ability to repair damage from low doses of radiation $(\sim 0.3 \mathrm{~Gy})$ over the course of several hours (Parkins et al. 1985), so most low-dose micro-CT scans should have limited biological impact, even when the same animals are longitudinally scanned over the course of a study. But, for higher-dose scans, longitudinal imaging can potentially lead to a cumulative dose that could affect biological function (particularly immune function and tumor response) and long-term health (Boone et al. 2004). Therefore, careful consideration must be made to determine the optimal imaging protocol for each individual application to minimize the effects of radiation dose on the experiment. With additional advances in micro-CT technology and reconstruction algorithms, radiation doses should further decrease, which will help to overcome radiation as a limitation of micro-CT imaging.

\subsection{Conclusions}

Micro-CT has become an extremely important tool in small animal research. Micro-CT produces non-invasive, 3D, highresolution anatomical images, which can provide a wealth of information about normal animal function and pathology. The increasing availability and low cost of micro-CT scanners (compared, for example, to MRI scanners) greatly increases the use and impact of micro-CT imaging on small animal studies. Radiation dose is a limitation in micro-CT imaging, but can be reduced with better technology and more sophisticated image reconstruction algorithms. Although X-ray micro-CT is limited by low tissue contrast, developments in contrast agent design show great promise for use in imaging a wide range of organ systems and pathologies. Additional new developments in spectral and phase contrast imaging will further improve the usefulness of micro-CT in acquiring 
functional and molecular information. This will greatly expand the potential applications for micro-CT in small animal research.

\section{Acknowledgements}

NIH/NIBIB national Biomedical Technology Resource Center (P41 EB015897); NIH National Cancer Institute (R01 CA196667). The author would like to thank Mrs Sally Zimney for editorial work.

\section{REFERENCES}

Almajdub, M., L. Magnier, L. Juillard, and M. Janier. 2008. Kidney volume quantification using contrast-enhanced in vivo X-ray micro-CT in mice. Contrast Media Mol Imaging 3(3), 120126. doi:10.1002/cmmi.238.

Almajdub, M., M. Nejjari, G. Poncet, L. Magnier, E. Chereul, C. Roche, and M. Janier. 2007. In-vivo high-resolution X-ray microtomography for liver and spleen tumor assessment in mice. Contrast Media Mol Imaging 2(2), 88-93.

Alric, C. et al. 2008. Gadolinium chelate coated gold nanoparticles as contrast agents for both X-ray computed tomography and magnetic resonance imaging. Journal of the American Chemical Society 130(18), 5908-5915. doi:10.1021/Ja078176p.

Anayama, T., T. Nakajima, M. Dunne, J. Zheng, C. Allen, B. Driscoll, D. Vines, S. Keshavjee, D. Jaffray, and K. Yasufuku. 2013. A novel minimally invasive technique to create a rabbit VX2 lung tumor model for nano-sized image contrast and interventional studies. PLoS One 8(6), e67355. doi:10.1371/ journal.pone.0067355.

Anderson, N. G. et al. 2010. Spectroscopic (multi-energy) CT distinguishes iodine and barium contrast material in MICE. Eur Radiol 20(9), 2126-2134. doi:10.1007/s00330-010-1768-9.

Annapragada, A. V., E. Hoffman, A. Divekar, E. Karathanasis, and K. B. Ghaghada. 2012. High-resolution CT vascular imaging using blood pool contrast agents. Methodist Debakey Cardiovasc J 8(1), 18-22.

Appleton, C. T., D. D. McErlain, V. Pitelka, N. Schwartz, S. M. Bernier, J. L. Henry, D. W. Holdsworth, and F. Beier. 2007. Forced mobilization accelerates pathogenesis: Characterization of a preclinical surgical model of osteoarthritis. Arthritis Res Ther 9(1), R13.

Aran, S., L. Daftari Besheli, M. Karcaaltincaba, R. Gupta, E. J. Flores, and H. H. Abujudeh. 2014. Applications of dualenergy CT in emergency radiology. AJR Am J Roentgenol 202(4), W314-W324. doi:10.2214/AJR.13.11682.

Artaechevarria, X. et al. 2011. Evaluation of micro-CT for emphysema assessment in mice: Comparison with non-radiological techniques. Eur Radiol 21(5), 954-962. doi:10.1007/ s00330-010-1982-5.

Ashton, J. R., N. Befera, D. Clark, Y. Qi, L. Mao, H. A. Rockman, G. A. Johnson, and C. T. Badea. 2014a. Anatomical and functional imaging of myocardial infarction in mice using micro-CT and eXIA 160 contrast agent. Contrast Media Mol Imaging 9(2), 161-168. doi:10.1002/cmmi.1557.

Ashton, J. R., D. P. Clark, E. J. Moding, K. Ghaghada, D. G. Kirsch, J. L. West, and C. T. Badea. 2014b. Dual-energy micro-CT functional imaging of primary lung cancer in mice using gold and iodine nanoparticle contrast agents: A validation study. PLoS One 9(2), e88129. doi:10.1371/journal.pone.0088129.
Aviv, H., S. Bartling, F. Kieslling, and S. Margel. 2009. Radiopaque iodinated copolymeric nanoparticles for X-ray imaging applications. Biomaterials 30(29), 5610-5616. doi:10.1016/j. biomaterials.2009.06.038.

Badea, C., B. Fubara, L. Hedlund, and G. A. Johnson. 2005. 4D micro-CT of the mouse heart. Molecular Imaging 4(2), $110-116$.

Badea, C., L. W. Hedlund, and G. A. Johnson. 2004a. Micro-CT with respiratory and cardiac gating. Med Phys 31(12), 3324-3329.

Badea, C., E. Schreibmann, and T. Fox. 2008a. A registration based approach foro $4 \mathrm{D}$ cardiac micro-CT using combined prospective and retrospective gating. Med Phys 35(4), 1170-1179.

Badea, C. T., K. K. Athreya, G. Espinosa, D. Clark, A. P. Ghafoori, Y. Li, D. G. Kirsch, G. A. Johnson, A. Annapragada, and K. B. Ghaghada. 2012. Computed tomography imaging of primary lung cancer in mice using a liposomal-iodinated contrast agent. Plos One 7(4), e34496. doi:10.1371/journal. pone. 0034496 .

Badea, C. T., K. Ghaghada, G. Espinosa, L. Strong, and A. Annapragada. 2011a. Multi-modality PET-CT imaging of breast cancer in an animal model using nanoparticle X-ray contrast agent and 18F-FDG. Medical Imaging 2011: Biomedical Applications in Molecular, Structural, and Functional Imaging 7965. doi:Artn 796511 Doi 10.1117/12.878024.

Badea, C. T., L. W. Hedlund, J. Cook, B. R. Berridge, and G. A. Johnson. 2011b. Micro-CT imaging assessment of dobutamineinduced cardiac stress in rats. Journal of Pharmacological and Toxicological Methods 63(1), 24-29. doi: 10.1016/j. vasen.2010.04.002.

Badea, C. T., L. W. Hedlund, M. De Lin, J. F. Boslego Mackel, and G. A. Johnson. 2006. Tumor imaging in small animals with a combined micro-CT/micro-DSA system using iodinated conventional and blood pool contrast agents. Contrast Media Mol Imaging 1(4), 153-164. doi:10.1002/cmmi.103.

Badea, C. T., L. W. Hedlund, and G. A. Johnson. 2004b. Micro-CT with respiratory and cardiac gating. Medical Physics 31(12), 3324-3329.

Badea, C. T., L. W. Hedlund, J. F. Mackel, L. Mao, H. A. Rockman, and G. A. Johnson. 2007. Cardiac micro-computed tomography for morphological and functional phenotyping of muscle LIM protein null mice. Mol Imaging 6(4), 261-268.

Badea, C. T., S. M. Johnston, Y. Qi, and G. A. Johnson. 2011c. 4D micro-CT for cardiac and perfusion applications with view under sampling. Phys Med Biol 56(11), 3351-3369. doi:10.1088/0031-9155/56/11/011.

Badea, C. T., E. Schreibmann, and T. Fox. 2008b. A registrationbased approach for cardiac micro-CT using combined prospective and retrospective gating. Medical Physics 35(4), 1170-1179.

Badea, C. T., A. W. Wetzel, N. Mistry, S. Pomerantz, D. Nave, and G. A. Johnson. 2008c. Left ventricle volume measurements in cardiac micro-CT: The impact of radiation dose and contrast agent. Comput Med Imaging Graph 32(3), 239-250.

Bartling, S. H., J. Dinkel, W. Stiller, M. Grasruck, I. Madisch, H. U. Kauczor, W. Semmler, R. Gupta, and F. Kiessling. 2008. Intrinsic respiratory gating in small-animal CT. European Radiology 18(7), 1375-1384. doi:10.1007/s00330-008-0903-3.

Bartling, S. H., W. Stiller, M. Grasruck, B. Schmidt, P. Peschke, W. Semmler, and F. Kiessling. 2007. Retrospective motion gating in small animal CT of mice and rats. Invest Radiol 42(10), 704-714. 
Bech, M., A. Tapfer, A. Velroyen, A. Yaroshenko, B. Pauwels, J. Hostens, P. Bruyndonckx, A. Sasov, and F. Pfeiffer. 2013. In-vivo dark-field and phase-contrast X-ray imaging. Scientific Reports 3, 3209, doi:10.1038/srep03209.

Beque, D., J. Nuyts, G. Bormans, P. Suetens, and P. Dupont. 2003. Characterization of pinhole SPECT acquisition geometry. IEEE Trans Med Imaging 22(5), 599-612. doi:10.1109/ TMI.2003.812258.

Bevins, N., J. Zambelli, K. Li, Z. Qi, and G.-H. Chen. 2011. Multicontrast X-ray computed tomography imaging using Talbot-Lau interferometry without phase stepping. Medical Physics 39(1), 424-428.

Bhavane, R., C. Badea, K. B. Ghaghada, D. Clark, D. Vela, A. Moturu, A. Annapragada, G. A. Johnson, J. T. Willerson, and A. Annapragada. 2013. Dual-energy computed tomography imaging of atherosclerotic plaques in a mouse model using a liposomal-iodine nanoparticle contrast agent. Circ Cardiovasc Imaging 6(2), 285-294. doi:10.1161/CIRCIM AGING.112.000119.

Boll, H., S. Bag, I. S. Nölte, T. Wilhelm, M. Kramer, C. Groden, U. Böcker, and M. A. Brockmann. 2011a. Double-contrast micro-CT colonoscopy in live mice. International Journal of Colorectal Disease 26(6), 721-727.

Boll, H., S. Nittka, F. Doyon, M. Neumaier, A. Marx, M. Kramer, C. Groden, and M. A. Brockmann. 2011b. Micro-CT based experimental liver imaging using a nanoparticulate contrast agent: A longitudinal study in mice. Plos ONE 6 (9):e25692. doi:ARTN e25692 10.1371/journal.pone.0025692.

Bongartz, T. et al. 2014. Dual-energy CT for the diagnosis of gout: An accuracy and diagnostic yield study. Ann Rheum Dis. 74(6):1072-7. June 2015. doi:10.1136/ annrheumdis-2013-205095.

Boone, J. M., G. M. Alexander, and J. A. Seibert. 1993. A fluoroscopy-based computed tomography scanner for small specimen research. Invest Radiol 28(6), 539-544.

Boone, J. M., O. Velazquez, and S. R. Cherry. 2004. Small-animal X-ray dose from micro-CT. Mol Imaging 3(3), 149-158.

Borg, J. S., M.-A. Vu, C. Badea, A. Badea, G. A. Johnson, and K. Dzirasa. 2015. Localization of metal electrodes in the intact rat brain using registration of $3 \mathrm{D}$ microcomputed tomography images to a magnetic resonance histology atlas. Eneuro 2(4):ENEURO.0017-15.2015. doi: https://doi.org/10.1523/ ENEURO.0017-15.2015.

Bornefalk, H. and M. Danielsson. 2010. Photon-counting spectral computed tomography using silicon strip detectors: A feasibility study. Phys Med Biol 55(7), 1999-2022. doi:10.1088/ 0031-9155/55/7/014.

Bourin, M., P. Jolliet, and F. Ballereau. 1997. An overview of the clinical pharmacokinetics of X-ray contrast media. Clin Pharmacokinet 32(3), 180-193.

Bouxsein, M. L., S. K. Boyd, B. A. Christiansen, R. E. Guldberg, K. J. Jepsen, and R. Muller. 2010. Guidelines for assessment of bone microstructure in rodents using micro-computed tomography. J Bone Miner Res 25(7), 1468-1486. doi:10.1002/jbmr.141.

Bravin, A., P. Coan, and P. Suortti. 2013. X-ray phase-contrast imaging: From pre-clinical applications towards clinics. Physics in Medicine and Biology 58(1), R1.

Brunke, O., K. Brockdorf, S. Drews, B. Müller, T. Donath, J. Herzen, and F. Beckmann. 2008. Comparison between X-ray tubebased and synchrotron radiation-based $\mu$ CT.70780U-70780U. Proc. of SPIE Vol. 7078, pp. 70780U-1. doi:10.1117/12.794789.
Campbell, M., E. H. M. Heijne, G. Meddeler, E. Pernigotti, and W. Snoeys. 1998. A readout chip for a $64 \times 64$ pixel matrix with 15-bit single photon counting. Nuclear Science, IEEE Transactions on 45(3), 751-753.

Cao, G., L. M. Burk, Y. Z. Lee, X. Calderon-Colon, S. Sultana, J. Lu, and O. Zhou. 2010. Prospective-gated cardiac micro-CT imaging of free-breathing mice using carbon nanotube field emission X-ray. Med Phys 37(10), 5306-5312.

Cao, G. et al. 2009. A dynamic micro-CT scanner based on a carbon nanotube field emission X-ray source. Phys Med Biol 54(8), 2323-2340.

Carlson, S. K., K. L. Classic, C. E. Bender, and S. J. Russell. 2007. Small animal absorbed radiation dose from serial microcomputed tomography imaging. Mol Imaging Biol 9(2), $78-82$.

Carril, M., I. Fernandez, J. Rodriguez, I. Garcia, and S. Penades. 2014. Gold-coated iron oxide glyconanoparticles for MRI, $\mathrm{CT}$, and US multimodal imaging. Particle \& Particle Systems Characterization 31(1), 81-87. doi:10.1002/ppsc. 201300239.

Cervenka, L., K. D. Mitchell, and L. G. Navar. 1999. Renal function in mice: Effects of volume expansion and angiotensin II. $J \mathrm{Am}$ Soc Nephrol 10(12), 2631-2636.

Chanda, N. et al. 2010. Bombesin functionalized gold nanoparticles show in vitro and in vivo cancer receptor specificity. Proc Natl Acad Sci USA 107(19), 8760-8765. doi:10.1073/ pnas.1002143107.

Chapman, D. W. Thomlinson, R. E. Johnston, D. Washburn, E. Pisano, N. Gmür, Z. Zhong, R. Menk, F. Arfelli, and D. Sayers. 1997. Diffraction enhanced X-ray imaging. Physics in Medicine and Biology 42(11), 2015.

Chen, F., E. B. Ehlerding, and W. Cai. 2014. Theranostic nanoparticles. J Nucl Med 55(12), 1919-1922. doi10.2967/ jnumed.114.146019.

Choi, E. J., G. Y. Jin, S. M. Bok, Y. M. Han, Y. S. Lee, M. J. Jung, and K. S. Kwon. 2014. Serial micro-CT assessment of the therapeutic effects of rosiglitazone in a bleomycin-induced lung fibrosis mouse model. Korean J Radiol 15(4), 448-455. doi:10.3348/kjr.2014.15.4.448.

Choquet, P., A. Calon, É. Breton, F. Beck, C. Domon-Dell, J.-N. Freund, and A. Constantinesco. 2007. Multiple-contrast X-ray micro-CT visualization of colon malformations and tumours in situ in living mice. Comptes Rendus Biologies 330(11), 821-827.

Chouker, A. et al. 2008. Comparison of Fenestra VC Contrastenhanced computed tomography imaging with gadopentetate dimeglumine and ferucarbotran magnetic resonance imaging for the in vivo evaluation of murine liver damage after ischemia and reperfusion. Invest Radiol 43(2), 77-91. doi:10.1097/ RLI.0b013e318155aa2e.

Chow, P. L., F. R. Rannou, and A. F. Chatziioannou. 2005. Attenuation correction for small animal PET tomographs. Phys Med Biol 50(8), 1837-1850. doi:10.1088/0031-9155/50/8/014.

Clark, D. P. and C. T. Badea. 2014. Spectral diffusion: An algorithm for robust material decomposition of spectral CT data. Phys Med Biol 59(21), 6445-6466. doi:10.1088/0031-9155/59/21/6445.

Clark, D. P., K. Ghaghada, E. J. Moding, D. G. Kirsch, and C. T. Badea. 2013. In vivo characterization of tumor vasculature using iodine and gold nanoparticles and dual energy micro-CT. Phys Med Biol 58(6), 1683-1704. doi:10.1088/00319155/58/6/1683. 
Cole,L.E., T. Vargo-Gogola, and R. K. Roeder. 2014. Bisphosphonatefunctionalized gold nanoparticles for contrast-enhanced X-ray detection of breast microcalcifications. Biomaterials 35(7), 2312-2321. doi:10.1016/j.biomaterials.2013.11.077.

Connor, D. M., E. D. Pisano, E. B. Cole, Z. Zhong, F. A. Dilmanian, C. A. Parham, A. Bravin, P. Coan, M. Fernandez, and J. Keyriläinen. 2011. Translation of synchrotron-based research into the clinic: Assessing the current clinical potential of diffraction enhanced imaging. Synchrotron Radiation News 24(2), 29-33.

Cormode, D. P., P. C. Naha, and Z. A. Fayad. 2014. Nanoparticle contrast agents for computed tomography: A focus on micelles. Contrast Media Mol Imaging 9(1), 37-52. doi:10.1002/ cmmi.1551.

Cormode, D. P. et al. 2010. Atherosclerotic plaque composition: Analysis with multicolor CT and targeted gold nanoparticles. Radiology 256(3), 774-782. doi:10.1148/radiol.10092473.

Coughlin, A. J., J. S. Ananta, N. Deng, I. V. Larina, P. Decuzzi, and J. L. West. 2014. Gadolinium-conjugated gold nanoshells for multimodal diagnostic imaging and photothermal cancer therapy. Small 10(3), 556-565. doi:10.1002/smll.201302217.

Cowan, C. M., T. Aghaloo, Y. F. Chou, B. Walder, X. Zhang, C. Soo, K. Ting, and B. Wu. 2007. MicroCT evaluation of threedimensional mineralization in response to BMP-2 doses in vitro and in critical sized rat calvarial defects. Tissue Eng 13(3), 501-512.

Criscione, J. M., L. W. Dobrucki, Z. W. Zhuang, X. Papademetris, M. Simons, A. J. Sinusas, and T. M. Fahmy. 2011. Development and application of a multimodal contrast agent for SPECT/ CT hybrid imaging. Bioconjug Chem 22(9), 1784-1792. doi:10.1021/bc200162r.

Curry, T., R. Kopelman, M. Shilo, and R. Popovtzer. 2014. Multifunctional theranostic gold nanoparticles for targeted CT imaging and photothermal therapy. Contrast Media Mol Imaging 9(1), 53-61. doi:10.1002/cmmi.1563.

Danila, D., E. Johnson, and P. Kee. 2013. CT imaging of myocardial scars with collagen-targeting gold nanoparticles. Nanomedicine. 9(7), 1067-1076. doi:10.1016/j.nano.2013.03.009.

David, C., T. Weitkamp, F. Pfeiffer, A. Diaz, J. Bruder, T. Rohbeck, A. Groso, O. Bunk, M. Stampanoni, and P. Cloetens. 2007. Hard X-ray phase imaging and tomography using a grating interferometer. Spectrochimica Acta Part B: Atomic Spectroscopy 62(6), 626-630.

David, V., N. Laroche, B. Boudignon, M. H. Lafage-Proust, C. Alexandre, P. Ruegsegger, and L. Vico. 2003. Noninvasive in vivo monitoring of bone architecture alterations in hindlimb-unloaded female rats using novel three-dimensional microcomputed tomography. J Bone Miner Res 18(9), $1622-1631$.

De Langhe, E., G. V. Velde, J. Hostens, U. Himmelreich, B. Nemery, F. P. Luyten, J. Vanoirbeek, and R. J. Lories. 2012. Quantification of lung fibrosis and emphysema in mice using automated micro-computed tomography. PLoS One 7(8), e43123. doi:10.1371/journal.pone.0043123.

de Vries, A., E. Custers, J. Lub, S. van den Bosch, K. Nicolay, and H. Grull. 2010. Block-copolymer-stabilized iodinated emulsions for use as CT contrast agents. Biomaterials 31(25), 6537-6544. doi:10.1016/j.biomaterials.2010.04.056.

Defrise, M. and R. Clack. 1995. Filtered backprojection reconstruction of combined parallel beam and cone beam SPECT data. Phys Med Biol 40(9), 1517-1537.
Defrise, M., C. Vanhove, and J. Nuyts. 2008. Perturbative refinement of the geometric calibration in pinhole SPECT. IEEE Trans Med Imaging 27(2), 204-214. doi:10.1109/TMI.2007.904687.

Desnoyers, L. R. et al. 2008. Targeting FGF19 inhibits tumor growth in colon cancer xenograft and FGF19 transgenic hepatocellular carcinoma models. Oncogene 27(1), 85-97. doi:10.1038/ sj.onc.1210623.

Diehl, K. H., R. Hull, D. Morton, R. Pfister, Y. Rabemampianina, D. Smith, J. M. Vidal, C. van de Vorstenbosch. Association European Federation of Pharmaceutical Industries, and Methods European Centre for the Validation of Alternative. 2001. A good practice guide to the administration of substances and removal of blood, including routes and volumes. J Appl Toxicol 21(1), 15-23.

Dinkel, J. et al. 2008. Intrinsic gating for small-animal computed tomography a robust ECG-less paradigm for deriving cardiac phase information and functional imaging. CirculationCardiovascular Imaging 1(3), 235-243. doi: 10.1161/ Circimaging.108.784702.

Drangova, M., N. L. Ford, S. A. Detombe, A. R. Wheatley, and D. W. Holdsworth. 2007. Fast retrospectively gated quantitative four-dimensional (4D) cardiac micro computed tomography imaging of free-breathing mice. Invest Radiol 42(2), 85-94.

Du, L. Y., J. Umoh, H. N. Nikolov, S. I. Pollmann, T. Y. Lee, and D. W. Holdsworth. 2007. A quality assurance phantom for the performance evaluation of volumetric micro-CT systems. Phys Med Biol 52(23), 7087-7108.

Dumas, A., M. Brigitte, M. F. Moreau, F. Chretien, M. F. Basle, and D. Chappard. 2009. Bone mass and microarchitecture of irradiated and bone marrow-transplanted mice: Influences of the donor strain. Osteoporos Int 20(3), 435-443. doi:10.1007/ s00198-008-0658-3.

Durkee, B. Y., S. R. Mudd, C. N. Roen, L. Clipson, M. A. Newton, J. P. Weichert, P. J. Pickhardt, and R. B. Halberg. 2008. Reproducibility of tumor volume measurement at microCT colonography in living mice. Academic Radiology 15(3), 334-341.

Eck, W., A. I. Nicholson, H. Zentgraf, W. Semmler, and S. Bartling. 2010. Anti-CD4-targeted gold nanoparticles induce specific contrast enhancement of peripheral lymph nodes in X-ray computed tomography of live mice. Nano Lett 10(7), 23182322. doi:10.1021/nl101019s.

Ekdawi, S. N., J. M. Stewart, M. Dunne, S. Stapleton, N. Mitsakakis, Y. N. Dou, D. A. Jaffray, and C. Allen. 2015. Spatial and temporal mapping of heterogeneity in liposome uptake and microvascular distribution in an orthotopic tumor xenograft model. J Control Release 207, 101-111. doi:10.1016/j. jconrel.2015.04.006.

Elbakri, I. A. and J. A. Fessler. 2002. Statistical image reconstruction for polyenergetic X-ray computed tomography. IEEE Trans Med Imaging 21, 89-99.

Epple, F. M., G. Potdevin, P. Thibault, S. Ehn, J. Herzen, A. Hipp, F. Beckmann, and F. Pfeiffer. 2013. Unwrapping differential $\mathrm{X}$-ray phase-contrast images through phase estimation from multiple energy data. Optics Express 21(24), 29101-29108.

Erathodiyil, N. and J. Y. Ying. 2011. Functionalization of inorganic nanoparticles for bioimaging applications. Accounts of Chemical Research 44(10), 925-935. doi:10.1021/Ar 2000327.

Feldkamp, L. A., L. C. Davis, and J. W. Kress. 1984. Practical conebeam algorithm. J. Opt. Soc. Am. 1(6), 612-619. 
Feldkamp, L. A., S. A. Goldstein, A. M. Parfitt, G. Jesion, and M. Kleerekoper. 1989. The direct examination of threedimensional bone architecture in vitro by computed tomography. J Bone Miner Res 4(1), 3-11.

Feng, G., B. Kong, J. Xing, and J. Chen. 2014. Enhancing multimodality functional and molecular imaging using glucose-coated gold nanoparticles. Clinical Radiology 69(11), 1105-1111. doi:10.1016/j.crad.2014.05.112.

Fiebig, T., H. Boll, G. Figueiredo, H. U. Kerl, S. Nittka, C. Groden, M. Kramer, and M. A. Brockmann. 2012. Three-dimensional in vivo imaging of the murine liver: A micro-computed tomography-based anatomical study. PLoS One 7(2), e31179. doi:10.1371/journal.pone.0031179.

Fivez, C. M., P. Wambacq, P. Suetens, and E. P. Schoeters. 1996. Influence of Heel effect and of nonuniformity of emitted spectra on dual-energy subtraction in computed radiography.

Ford, N. L., H. N. Nikolov, C. J. Norley, M. M. Thornton, P. J. Foster, M. Drangova, and D. W. Holdsworth. 2005. Prospective respiratory-gated micro-CT of free breathing rodents. Med Phys 32(9), 2888-2898.

Ford, N. L., A. R. Wheatley, D. W. Holdsworth, and M. Drangova. 2007. Optimization of a retrospective technique for respiratory-gated high speed micro-CT of free-breathing rodents. Phys Med Biol 52(19), 5749-5769.

Fu, J., S. Schleede, R. Tan, L. Chen, M. Bech, K. Achterhold, M. Gifford, R. Loewen, R. Ruth, and F. Pfeiffer. 2013. An algebraic iterative reconstruction technique for differential X-ray phase-contrast computed tomography. Zeitschrift für Medizinische Physik 23(3), 186-193.

Fu, J., M. Willner, L. Chen, R. Tan, K. Achterhold, M. Bech, J. Herzen, D. Kunka, J. Mohr, and F. Pfeiffer. 2014. Helical differential X-ray phase-contrast computed tomography. Physica Medica 30(3), 374-379.

Galperin, A., D. Margel, J. Baniel, G. Dank, H. Biton, and S. Margel. 2007. Radiopaque iodinated polymeric nanoparticles for X-ray imaging applications. Biomaterials 28(30), 4461-4468. doi:10.1016/j.biomaterials.2007.06.032.

Ghaghada, K. B., C. T. Badea, L. Karumbaiah, N. Fettig, R. V. Bellamkonda, G. A. Johnson, and A. Annapragada. 2011. Evaluation of tumor microenvironment in an animal model using a nanoparticle contrast agent in computed tomography imaging. Academic Radiology 18(1), 20-30.

Ghann, W. E., O. Aras, T. Fleiter, and M. C. Daniel. 2012. Syntheses and characterization of lisinopril-coated gold nanoparticles as highly stable targeted CT contrast agents in cardiovascular diseases. Langmuir 28(28), 10398-10408. doi:10.1021/la301694q.

Goertzen, A. L., A. K. Meadors, R. W. Silverman, and S. R. Cherry. 2002. Simultaneous molecular and anatomical imaging of the mouse in vivo. Phys Med Biol 47(24), 4315-4328.

Goertzen, A. L., V. Nagarkar, R. A. Street, M. J. Paulus, J. M. Boone, and S. R. Cherry. 2004. A comparison of X-ray detectors for mouse CT imaging. Phys Med Biol 49(23), 5251-5265.

Gordon, R., R. Bender, and G. T. Herman. 1970. Algebraic reconstruction techniques (ART) for three-dimensional electron microscopy and X-ray photography. J. Theor. Biol. 29, 471-481.

Graham, K. C., N. L. Ford, L. T. MacKenzie, C. O. Postenka, A. C. Groom, I. C. MacDonald, D. W. Holdsworth, M. Drangova, and A. F. Chambers. 2008. Noninvasive quantification of tumor volume in preclinical liver metastasis models using contrast-enhanced X-ray computed tomography. Invest Radiol 43(2), 92-99.
Grider, A., Jr. and L. C. Erway. 1986. Intestinal metallothionein in lethal-milk mice with systemic zinc deficiency. Biochem Genet 24(7-8), 635-642.

Guerrero, T., R. Castillo, K. Sanders, R. Price, R. Komaki, and D. Cody. 2006. Novel method to calculate pulmonary compliance images in rodents from computed tomography acquired at constant pressures. Phys Med Biol 51(5), 1101-1112.

Guldberg, R. E., A. S. Lin, R. Coleman, G. Robertson, and C. Duvall. 2004. Microcomputed tomography imaging of skeletal development and growth. Birth Defects Res C Embryo Today 72(3), 250-259.

Guo, X., S. M. Johnston, Y. Qi, G. A. Johnson, and C. T. Badea. 2012a. 4D micro-CT using fast prospective gating. Phys Med Biol 57(1), 257-271. doi:10.1088/0031-9155/57/1/257.

Guo, X. L., S. M. Johnston, G. A. Johnson, and C. T. Badea. 2012b. A comparison of sampling strategies for dual energy microCT. Medical Imaging 2012: Physics of Medical Imaging 8313. doi:Artn 831332 Doi 10.1117/12.911548.

Hainfeld, J. F., F. A. Dilmanian, D. N. Slatkin, and H. M. Smilowitz. 2008. Radiotherapy enhancement with gold nanoparticles. J Pharm Pharmacol 60(8), 977-985. doi:10.1211/ jpp.60.8.0005.

Hainfeld, J. F., F. A. Dilmanian, Z. Zhong, D. N. Slatkin, J. A. Kalef-Ezra, and H. M. Smilowitz. 2010. Gold nanoparticles enhance the radiation therapy of a murine squamous cell carcinoma. Phys Med Biol 55(11), 3045-3059. doi:10.1088/0031-9155/55/11/004

Hainfeld, J. F., L. Lin, D. N. Slatkin, F. A. Dilmanian, T. M. Vadas, and H. M. Smilowitz. 2014. Gold nanoparticle hyperthermia reduces radiotherapy dose. Nanomedicine 10(8), 1609-1617. doi:10.1016/j.nano.2014.05.006.

Hainfeld, J. F., M. J. O’Connor, F. A. Dilmanian, D. N. Slatkin, D. J. Adams, and H. M. Smilowitz. 2011. Micro-CT enables microlocalisation and quantification of Her2-targeted gold nanoparticles within tumour regions. Br J Radiol 84(1002), 526-533. doi:10.1259/bjr/42612922.

Hainfeld, J. F., D. N. Slatkin, T. M. Focella, and H. M. Smilowitz. 2006. Gold nanoparticles: A new X-ray contrast agent. $\mathrm{Br} J$ Radiol 79(939):248-253. doi:10.1259/bjr/13169882.

Hainfeld, J. F., D. N. Slatkin, and H. M. Smilowitz. 2004. The use of gold nanoparticles to enhance radiotherapy in mice. Phys Med Biol 49(18), N309-N315.

Hainfeld, J. F., H. M. Smilowitz, M. J. O’Connor, F. A. Dilmanian, and D. N. Slatkin. 2013. Gold nanoparticle imaging and radiotherapy of brain tumors in mice. Nanomedicine (Lond) 8(10), 1601-1609. doi:10.2217/nnm.12.165.

Hallouard, F., N. Anton, P. Choquet, A. Constantinesco, and T. Vandamme. 2010. Iodinated blood pool contrast media for preclinical X-ray imaging applications-a review. Biomaterials 31(24), 6249-6268. doi:10.1016/j. biomaterials.2010.04.066.

Hallouard, F., S. Briancon, N. Anton, X. Li, T. Vandamme, and H. Fessi. 2013. Iodinated nano-emulsions as contrast agents for preclinical X-ray imaging: Impact of the free surfactants on the pharmacokinetics. Eur J Pharm Biopharm 83(1), 54-62. doi:10.1016/j.ejpb.2012.09.003.

Hedlund, L. W. and G. A. Johnson. 2002. Mechanical ventilation for imaging the small animal lung. ILAR J 43(3), 159-174.

Herzen, J. M. S. Willner, A. A. Fingerle, P. B. Noël, T. Köhler, E. Drecoll, E. J. Rummeny, and F. Pfeiffer. 2014. Imaging liver lesions using grating-based phase-contrast computed 
tomography with bi-lateral filter post-processing. PloS one 9(1), e83369.

Ho, S. T. and D. W. Hutmacher. 2006. A comparison of micro CT with other techniques used in the characterization of scaffolds. Biomaterials 27(8), 1362-1376. doi: 10.1016/j. biomaterials.2005.08.035.

Holdsworth, D. W., M. Drangova, and A. Fenster. 1993. A highresolution XRII-based quantitative volume $\mathrm{CT}$ scanner. Med Phys 20(2 Pt 1), 449-462.

Holdsworth, D. W. and M. M. Thornton. 2002. Micro-CT in small animal and specimen imaging. Trends in Biotechnology 20(8), S34-S39.

Hori, Y. et al. 2008. Periodic analysis of urethane-induced pulmonary tumors in living $\mathrm{A} / \mathrm{J}$ mice by respiration-gated X-ray microcomputed tomography. Cancer Sci 99(9), 1774-1777. doi:10.1111/j.1349-7006.2008.00889.x.

Hsu, J. T., Y. J. Chen, J. T. Ho, H. L. Huang, S. P. Wang, F. C. Cheng, J. Wu, and M. T. Tsai. 2014. A comparison of micro-CT and dental CT in assessing cortical bone morphology and trabecular bone microarchitecture. PLoS One 9(9), e107545. doi:10.1371/journal.pone.0107545.

Huang, P. et al. 2011. Folic acid-conjugated silica-modified gold nanorods for X-ray/CT imaging-guided dual-mode radiation and photo-thermal therapy. Biomaterials 32(36), 9796-9809. doi:10.1016/j.biomaterials.2011.08.086.

Hwang, A. B. and B. H. Hasegawa. 2005. Attenuation correction for small animal SPECT imaging using X-ray CT data. Med Phys 32(9), 2799-2804.

Jakab, G., A. Racz, P. Major, T. Buekki, and G. Nemeth. 2009. Fully GPU based real time corrections and reconstruction for cone beam micro CT. In 2009 IEEE Nuclear Science Symposium Conference Record, Vols 1-5, Ed. B. Yu, pp. 4068-4071.

Jakhmola, A., N. Anton, and T. F. Vandamme. 2012. Inorganic nanoparticles based contrast agents for X-ray computed tomography. Adv Healthc Mater 1(4), 413-431. doi:10.1002/ adhm.201200032.

Jepperson, M. A., J. G. Cernigliaro, D. Sella, E. Ibrahim, D. D. Thiel, S. Leng, and W. E. Haley. 2013. Dual-energy CT for the evaluation of urinary calculi: Image interpretation, pitfalls and stone mimics. Clin Radiol 68(12), e707-e714. doi:10.1016/j. crad.2013.07.012.

Jeremic, B., A. R. Aguerri, and N. Filipovic. 2013. Radiosensitization by gold nanoparticles. Clinical \& Translational Oncology 15(8), 593-601. doi: 10.1007/s12094-013-1003-7.

Jiang, S. D., L. S. Jiang, and L. Y. Dai. 2006. Spinal cord injury causes more damage to bone mass, bone structure, biomechanical properties and bone metabolism than sciatic neurectomy in young rats. Osteoporos Int 17(10), 1552-1561. doi:10.1007/s00198-006-0165-3.

Johnson, K. A. 2007. Imaging techniques for small animal imaging models of pulmonary disease: Micro-CT. Toxicologic Pathology 35(1), 59-64. doi:10.1080/01926230601184262.

Johnston, S. M., G. A. Johnson, and C. T. Badea. 2010a. GPU-based iterative reconstruction with total variation minimization for micro-CT. Proc. SPIE 7622, 762238.

Johnston, S. M., B. A. Perez, D. G. Kirsch, and C. T. Badea. 2010b. Phase-selective image reconstruction of the lungs in small animals using Micro-CT. Medical Imaging 2010: Physics of Medical Imaging 7622. doi:Artn 76223g Doi $10.1117 / 12.844359$.
Jokerst, J. V., T. Lobovkina, R. N. Zare, and S. S. Gambhir. 2011. Nanoparticle PEGylation for imaging and therapy. Nanomedicine (Lond) 6(4), 715-728. doi:10.2217/nnm.11.19.

Kachelriess, M., D. A. Sennst, W. Maxlmoser, and W. A. Kalender. 2002. Kymogram detection and kymogram-correlated image reconstruction from subsecond spiral computed tomography scans of the heart. Medical Physics 29(7), 1489-1503. doi:10.1118/1.1487861.

Kak, A. C. and M. Slaney. 1988. Principles of Computerized Tomographic Imaging. IEEE Press, New York.

Kalender, W. A. and Y. Kyriakou. 2007. Flat-detector computed tomography (FD-CT). Eur Radiol 17(11), 2767-2779. doi:10.1007/s00330-007-0651-9.

Karolczak, M., S. Schaller, K. Engelke, A. Lutz, U. Taubenreuther, K. Wiesent, and W. Kalender. 2001. Implementation of a conebeam reconstruction algorithm for the single-circle source orbit with embedded misalignment correction using homogeneous coordinates. Med Phys 28(10), 2050-2069.

Key, J. and J. F. Leary. 2014. Nanoparticles for multimodal in vivo imaging in nanomedicine. International Journal of Nanomedicine 9, 711-726. doi: 10.2147/Ijn.S53717.

Kiessling, F. et al. 2004. Volumetric computed tomography (VCT): A new technology for noninvasive, high-resolution monitoring of tumor angiogenesis. Nat Med 10(10), 1133-1138.

Kim, H. W., Q. Y. Cai, H. Y. Jun, K. S. Chon, S. H. Park, S. J. Byun, M. S. Lee, J. M. Oh, H. S. Kim, and K. H. Yoon. 2008. Micro-CT imaging with a hepatocyte-selective contrast agent for detecting liver metastasis in living mice. Acad Radiol 15(10), 1282-1290. doi:10.1016/j.acra.2008.03.021.

Kindlmann, G. L., D. M. Weinstein, G. M. Jones, C. R. Johnson, M. R. Capecchi, and C. Keller. 2005. Practical vessel imaging by computed tomography in live transgenic mouse models for human tumors. Mol Imaging 4(4), 417-424.

Kinney, J. H., N. E. Lane, and D. L. Haupt. 1995. In vivo, threedimensional microscopy of trabecular bone. J Bone Miner Res 10(2), 264-270.

Kirsch, D. G. et al. 2010. Imaging primary lung cancers in mice to study radiation biology. International Journal of Radiation Oncology, Biology, Physics 76(4), 973-977. doi:10.1016/j. ijrobp.2009.11.038.

Köhler, T., B. Brendel, and E. Roessl. 2011. Iterative reconstruction for differential phase contrast imaging using spherically symmetric basis functions. Medical Physics 38(8), $4542-4545$.

Krause, W., J. Leike, A. Sachse, and G. Schuhmann-Giampieri. 1993. Characterization of iopromide liposomes. Invest Radiol 28(11), 1028-1032.

Kuntz, J., J. Dinkel, S. Zwick, T. Bauerle, M. Grasruck, F. Kiessling, R. Gupta, W. Semmler, and S. H. Bartling. 2010. Fully automated intrinsic respiratory and cardiac gating for small animal CT. Phys Med Biol 55(7), 2069-2085. doi:10.1088/0031-9155/55/7/018.

Laib, A., O. Barou, L. Vico, M. H. Lafage-Proust, C. Alexandre, and P. Rugsegger. 2000. 3D micro-computed tomography of trabecular and cortical bone architecture with application to a rat model of immobilisation osteoporosis. Med Biol Eng Comput 38(3), 326-332.

Laib, A., J. L. Kumer, S. Majumdar, and N. E. Lane. 2001. The temporal changes of trabecular architecture in ovariectomized rats assessed by MicroCT. Osteoporosis International 12(11), 936-941. doi:10.1007/s001980170022. 
Langer, M., P. Cloetens, J.-P. Guigay, and F. Peyrin. 2008. Quantitative comparison of direct phase retrieval algorithms in in-line phase tomography. Medical Physics 35(10), 4556-4566.

Langheinrich, A. C. and E. L. Ritman. 2006. Quantitative imaging of microvascular permeability in a rat model of lipopolysaccharide-induced sepsis: Evaluation using cryostatic microcomputed tomography. Invest Radiol 41(8), 645-650. doi: 10.1097/01.rli.0000227494.17444.64.

Lee, C. L., H. Min, N. Befera, D. Clark, Y. Qi, S. Das, G. A. Johnson, C. T. Badea, and D. G. Kirsch. 2014. Assessing cardiac injury in mice with dual energy-MicroCT, 4D-MicroCT, and MicroSPECT imaging after partial heart irradiation. Int J Radiat Oncol Biol Phys 88(3), 686-693. doi:10.1016/j. ijrobp.2013.11.238.

Lee, S. C., H. K. Kim, I. K. Chun, M. H. Cho, S. Y. Lee, and M. H. Cho. 2003. A flat-panel detector based micro-CT system: Performance evaluation for small-animal imaging. Phys Med Biol 48(24):4173-4185.

Lee, S. W., P. Padmanabhan, P. Ray, S. S. Gambhir, T. Doyle, C. Contag, S. B. Goodman, and S. Biswal. 2009. Stem cellmediated accelerated bone healing observed with in vivo molecular and small animal imaging technologies in a model of skeletal injury. Journal of Orthopaedic Research 27(3), 295-302. doi:10.1002/jor.20736.

Li, M., A. Jirapatnakul, A. Biancardi, M. L. Riccio, R. S. Weiss, and A. P. Reeves. 2013a. Growth pattern analysis of murine lung neoplasms by advanced semi-automated quantification of micro-CT images. PLoS One 8(12), e83806. doi:10.1371/ journal.pone.0083806.

Li, R., D. J. Stewart, H. P. Von Schroeder, E. S. Mackinnon, and E. H. Schemitsch. 2009. Effect of cell-based VEGF gene therapy on healing of a segmental bone defect. Journal of Orthopaedic Research 27(1), 8-14. doi:10.1002/jor.20658.

Li, X., N. Anton, G. Zuber, and T. Vandamme. 2014. Contrast agents for preclinical targeted X-ray imaging. Adv Drug Deliv Rev 76, 116-133. doi:10.1016/j.addr.2014.07.013.

Li, X., N. Anton, G. Zuber, M. Zhao, N. Messaddeq, F. Hallouard, H. Fessi, and T. F. Vandamme. 2013b. Iodinated alphatocopherol nano-emulsions as non-toxic contrast agents for preclinical X-ray imaging. Biomaterials 34(2), 481-491. doi:10.1016/j.biomaterials.2012.09.026.

Li, X. F., P. Zanzonico, C. C. Ling, and J. O'Donoghue. 2006. Visualization of experimental lung and bone metastases in live nude mice by X-ray micro-computed tomography. Technol Cancer Res Treat 5(2), 147-155.

Li, X. M., L. Wang, Y. B. Fan, Q. L. Feng, and F. Z. Cui. 2012. Biocompatibility and Toxicity of Nanoparticles and Nanotubes. Journal of Nanomaterials 2012:6, Artn 548389. doi:10.1155/2012/548389.

Liang, H., Y. Yang, K. Yang, Y. Wu, J. M. Boone, and S. R. Cherry. 2007. A microPET/CT system for in vivo small animal imaging. Phys Med Biol 52(13), 3881-3894.

Lin, C. Y., R. M. Schek, A. S. Mistry, X. Shi, A. G. Mikos, P. H. Krebsbach, and S. J. Hollister. 2005. Functional bone engineering using ex vivo gene therapy and topology-optimized, biodegradable polymer composite scaffolds. Tissue Eng 11(9-10), 1589-1598. doi:10.1089/ten.2005.11.1589.

Lin, M. D., L. Ning, C. T. Badea, N. N. Mistry, Y. Qi, and G. A. Johnson. 2008. A high precision contrast injector for small animal X-ray digital subtraction angiography. IEEE Transactions on Biomedical Engineering 2008(3), 1082-1091.
Liu, Y., Ashton, J.R., Moding, E.J., Yuan. H., Register. J.K., Fales, A.M., and Choi, J. 2015. A plasmonic gold nanostar theranostic probe for in vivo tumor imaging and photothermal therapy. Theranostics 5(9), 946-960.

Lu, Z. R. 2014. Theranostics: Fusion of therapeutics and diagnostics. Pharmaceutical Research 31(6), 1355-1357. doi:10.1007/ s11095-014-1343-1.

Maeda, H. 2001. The enhanced permeability and retention (EPR) effect in tumor vasculature: The key role of tumor-selective macromolecular drug targeting. Adv Enzyme Regul 41, 189-207.

Maeda, H., J. Wu, T. Sawa, Y. Matsumura, and K. Hori. 2000. Tumor vascular permeability and the EPR effect in macromolecular therapeutics: A review. Journal of Controlled Release 65(1-2), 271-284. doi:10.1016/s0168-3659(99)00248-5.

Marin, D., D. T. Boll, A. Mileto, and R. C. Nelson. 2014. State of the art: Dual-energy CT of the abdomen. Radiology 271(2), 327-342. doi:10.1148/radiol.14131480.

McErlain, D. D., C. T. Appleton, R. B. Litchfield, V. Pitelka, J. L. Henry, S. M. Bernier, F. Beier, and D. W. Holdsworth. 2008. Study of subchondral bone adaptations in a rodent surgical model of $\mathrm{OA}$ using in vivo micro-computed tomography. Osteoarthritis Cartilage 16(4), 458-469.

Meinel, F. G. et al. 2014. Lung tumors on multimodal radiographs derived from grating-based X-ray imaging - A feasibility study. Physica Medica 30(3), 352-357. doi: 10.1016/j. ejmp.2013.11.001.

Mileto, A., D. Marin, R. C. Nelson, G. Ascenti, and D. T. Boll. 2014. Dual energy MDCT assessment of renal lesions: An overview. Eur Radiol 24(2), 353-362. doi:10.1007/s00330-013-3030-8.

Moding, E. J., D. P. Clark, Y. Qi, Y. Li, Y. Ma, K. Ghaghada, G. A. Johnson, D. G. Kirsch, and C. T. Badea. 2013. Dualenergy micro-computed tomography imaging of radiationinduced vascular changes in primary mouse sarcomas. Int J Radiat Oncol Biol Phys 85(5), 1353-1359. doi:10.1016/j. ijrobp.2012.09.027.

Moghimi, S. M., A. C. Hunter, and J. C. Murray. 2001. Longcirculating and target-specific nanoparticles: Theory to practice. Pharmacol Rev 53(2), 283-318.

Momose, A. 2005. Recent advances in X-ray phase imaging. Japanese Journal of Applied Physics 44(9R), 6355.

Montet, X., C. M. Pastor, J. P. Vallee, C. D. Becker, A. Geissbuhler, D. R. Morel, and P. Meda. 2007. Improved visualization of vessels and hepatic tumors by micro-computed tomography (CT) using iodinated liposomes. Invest Radiol 42(9), 652-658.

Mukundan, S., K. B. Ghaghada, C. T. Badea, L. W. Hedlund, G. A. Johnson, J. M. Provenzale, R. V. Bellamkonda, and A. Annapragada. 2006. A nanoscale, liposomal contrast agent for preclincal microct imaging of the mouse. AJR 186, 300-307.

Munoz-Barrutia, A., M. Ceresa, X. Artaechevarria, L. M. Montuenga, and C. Ortiz-de-Solorzano. 2012. Quantification of lung damage in an elastase-induced mouse model of emphysema. Int $J$ Biomed Imaging 2012, 734734. doi:10.1155/2012/734734.

Munro, P. R. T., K. Ignatyev, R. D. Speller, and A. Olivo. 2012. Phase and absorption retrieval using incoherent X-ray sources. Proceedings of the National Academy of Sciences 109(35), 13922-13927.

Nahrendorf, M., C. Badea, L. W. Hedlund, J. L. Figueiredo, D. E. Sosnovik, G. A. Johnson, and R. Weissleder. 2007. Highresolution imaging of murine myocardial infarction with delayed-enhancement cine micro-CT. Am J Physiol Heart Circ Physiol 292(6), H3172-8. 
Namasivayam, S., M. K. Kalra, W. E. Torres, and W. C. Small. 2006. Adverse reactions to intravenous iodinated contrast media: An update. Curr Probl Diagn Radiol 35(4), 164-169. doi:10.1067/j.cpradiol.2006.04.001.

Namati, E., D. Chon, J. Thiesse, E. A. Hoffman, J. de Ryk, A. Ross, and G. McLennan. 2006. In vivo micro-CT lung imaging via a computer-controlled intermittent iso-pressure breath hold (IIBH) technique. Phys Med Biol 51(23), 6061-6075.

Namati, E., J. Thiesse, J. C. Sieren, A. Ross, E. A. Hoffman, and G. McLennan. 2010. Longitudinal assessment of lung cancer progression in the mouse using in vivo micro-CT imaging. Med Phys 37(9), 4793-4805.

Nilchian, M. and M. Unser. 2012. Differential phase-contrast X-ray computed tomography: From model discretization to image reconstruction. Paper read at Biomedical Imaging (ISBI), 2012, 9th IEEE International Symposium on Biomedical Imaging (ISBI) 90-93. doi: 10.1109/ISBI.2012.6235491.

Noo, F., R. Clackdoyle, C. Mennessier, T. A. White, and T. J. Roney. 2000. Analytic method based on identification of ellipse parameters for scanner calibration in cone-beam tomography. Phys Med Biol 45(11), 3489-3508.

Ohana, M., M. Y. Jeung, A. Labani, S. E. Ghannudi, and C. Roy. 2014. Thoracic dual energy CT: Acquisition protocols, current applications and future developments. Diagn Interv Imaging 95(11), 1017-1026. doi:10.1016/j.diii.2014.01.001.

Pan, D. et al. 2010. Computed tomography in color: Nanok-enhanced Spectral CT molecular imaging. Angewandte ChemieInternational Edition 49(50), 9635-9639. doi:10.1002/ anie. 201005657.

Pan, D., C. O. Schirra, A. Senpan, A. H. Schmieder, A. J. Stacy, E. Roessl, A. Thran, S. A. Wickline, R. Proska, and G. M. Lanza. 2012. An early investigation of ytterbium nanocolloids for selective and quantitative multicolor spectral CT imaging. ACS Nano 6(4), 3364-3370. doi:10.1021/nn300392x.

Pandit, P., S. M. Johnston, Y. Qi, J. Story, R. Nelson, and G. A. Johnson. 2013. The utility of micro-CT and MRI in the assessment of longitudinal growth of liver metastases in a preclinical model of colon carcinoma. Acad Radiol 20(4), 430-439. doi:10.1016/j.acra.2012.09.030.

Panetta, D., N. Belcari, G. Baldazzi, C. Carpentieri, E. Cicalini, A. Del Guerra, J. Fogli, M. Quattrocchi, V. Rosso, and A. Stefanini. 2007. Characterization of a high-resolution CT scanner prototype for small animals. Nuovo Cimento Della Societa Italiana Di Fisica B-General Physics Relativity Astronomy and Mathematical Physics and Methods 122(6-7), 739-747. doi:10.1393/ncb/i2007-10417-4.

Panetta, D., N. Belcari, A. Del Guerra, and S. Moehrs. 2008. An optimization-based method for geometrical calibration in cone-beam CT without dedicated phantoms. Phys Med Biol 53(14), 3841-3861. doi:10.1088/0031-9155/53/14/009.

Park, J. et al. 2015. Multifunctional hollow gold nanoparticles designed for triple combination therapy and CT imaging. $J$ Control Release 207, 77-85. doi:10.1016/j.jconrel.2015.04.007.

Parkins, C. S., J. F. Fowler, R. L. Maughan, and M. J. Roper. 1985. Repair in mouse lung for up to 20 fractions of $\mathrm{X}$ rays or neutrons. Br J Radiol 58(687), 225-241.

Parkinson, C. R. and A. Sasov. 2008. High-resolution nondestructive 3D interrogation of dentin using X-ray nanotomography. Dent Mater 24(6), 773-777. doi:10.1016/j.dental. 2007.09.003.
Paul, J., T. J. Vogl, and E. C. Mbalisike. 2014. Oncological applications of dual-energy computed tomography imaging. J Comput Assist Tomogr 38(6), 834-842. doi:10.1097/ RCT.0000000000000133.

Paulus, M. J., S. S. Gleason, M. E. Easterly, and C. J. Foltz. 2001. A review of high-resolution X-ray computed tomography and other imaging modalities for small animal research. Lab Anim (NY) 30(3), 36-45.

Paulus, M. J., S. S. Gleason, S. J. Kennel, P. R. Hunsicker, and D. K. Johnson. 2000. High resolution X-ray computed tomography: An emerging tool for small animal cancer research. Neoplasia 2(1-2), 62-70.

Pauwels, B., P. Bruyndonckxa, X. Liua, A. Tapferb, A. Velroyenb, A. Yaroshenkob, M. Bechb, F. Pfeifferb, and A. Sasova. 2012. First small-animal in-vivo phase-contrast micro-CT scanner. SPIE Optical Engineering +Applications 8506, 85060J-85060J-8.

Perez, B. A., A. P. Ghafoori, S. M. Johnston, L. B. Jeffords, Y. Kim, C. T. Badea, G. A. Johnson, and D. G. Kirsch. 2009. Dissecting the mechanism of tumor response to radiation therapy with primary lung cancers in mice. International Journal of Radiation Oncology* Biology* Physics 75(3), S537-S537.

Perez, B. A. et al. 2013. Assessing the radiation response of lung cancer with different gene mutations using genetically engineered mice. Front Oncol 3, 72. doi:10.3389/fonc.2013.00072.

Petersein, J., B. Franke, X. Fouillet, and B. Hamm. 1999. Evaluation of liposomal contrast agents for liver $\mathrm{CT}$ in healthy rabbits. Invest Radiol 34(6), 401-409.

Pfeiffer, F., T. Weitkamp, O. Bunk, and C. David. 2006. Phase retrieval and differential phase-contrast imaging with lowbrilliance X-ray sources. Nature Physics 2(4), 258-261.

Pickhardt, P. J., R. B. Halberg, A. J. Taylor, B.Y. Durkee, J. Fine, F. T. Lee, and J. P. Weichert. 2005. Microcomputed tomography colonography for polyp detection in an in vivo mouse tumor model. Proceedings of the National Academy of Sciences of the United States of America 102(9), 3419-3422.

Postnov, A. A., K. Meurrens, H. Weiler, D. Van Dyck, H. Xu, P. Terpstra, and N. M. De Clerck. 2005. In vivo assessment of emphysema in mice by high resolution X-ray microtomography. J Microsc 220(Pt 1), 70-75.

Rajagopal, S., J. Kovacs, C. Badea, G.A. Johnson, H. A. Rockman, C. A. Piantadosi, and R. J. Lefkowitz. 2011. Beta-arrestins regulate signaling by bone morphogenetic protein type II receptor in pulmonary arterial hypertension. Journal of the American College of Cardiology 14(57):E2046.

Reuveni, T., M. Motiei, Z. Romman, A. Popovtzer, and R. Popovtzer. 2011. Targeted gold nanoparticles enable molecular CT imaging of cancer: An in vivo study. Int J Nanomedicine 6, 28592864. doi:10.2147/IJN.S25446.

Ritman, E. L. 2004. Micro-computed tomography-current status and developments. Annu Rev Biomed Eng 6, 185-208.

Ritman, E. L., S. M. Jorgensen, P. E. Beighley, P. J. Thomas, J. H. Dunsmuir, J. C. Romero, R. T. Turner, and M. E. Bolander. 1997. Synchrotron-based micro-CT of in-situ biological basic functional units and their integration. Proc. SPIE 3149, Developments in X-Ray Tomography, 13 (October 24, 1997); doi:10.1117/12.279356.

Roa, W., Y. P. Xiong, J. Chen, X. Y. Yang, K. Song, X. H. Yang, B. H. Kong, J. Wilson, and J. Z. Xing. 2012. Pharmacokinetic and toxicological evaluation of multi-functional thiol-6-fluoro6-deoxy-D-glucose gold nanoparticles in vivo. Nanotechnology 23(37) Artn 375101. doi:10.1088/0957-4484/23/37/375101. 
Roessl, E., D. Cormode, B. Brendel, K. J. Engel, G. Martens, A. Thran, Z. Fayad, and R. Proksa. 2011. Preclinical spectral computed tomography of gold nano-particles. Nuclear Instruments \& Methods in Physics Research Section a-Accelerators Spectrometers Detectors and Associated Equipment 648, S259-S264. doi:10.1016/j.nima.2010.11.072.

Ross, W., D. D. Cody, and J. D. Hazle. 2006. Design and performance characteristics of a digital flat-panel computed tomography system. Med Phys 33(6), 1888-1901.

Rothe, J. H. et al. 2015. Time course of contrast enhancement by micro-CT with dedicated contrast agents in normal mice and mice with hepatocellular carcinoma: Comparison of one iodinated and two nanoparticle-based agents. Acad Radiol 22(2), 169-178. doi:10.1016/j.acra.2014.07.022.

Rudin, L. I., S. Osher, and E. Fatemi. 1992. Nonlinear total variation based noise removal algorithms. Physica D 60, 259-268.

Rudyanto, R. D., G. Bastarrika, G. de Biurrun, J. Agorreta, L. M. Montuenga, C. Ortiz-de-Solorzano, and A. Munoz-Barrutia. 2013. Individual nodule tracking in micro-CT images of a longitudinal lung cancer mouse model. Med Image Anal 17(8), 1095-1105. doi:10.1016/j.media.2013.07.002.

Ryu, J. H., S. Lee, S. Son, S. H. Kim, J. F. Leary, K. Choi, and I. C. Kwon. 2014. Theranostic nanoparticles for future personalized medicine. Journal of Controlled Release 190, 477-484. doi:10.1016/j.jconrel.2014.04.027.

Saito, S. and K. Murase. 2012. Detection and early phase assessment of radiation-induced lung injury in mice using micro-CT. PLoS One 7(9), e45960. doi:10.1371/journal.pone.0045960.

Samei, E., R. S. Saunders, C. T. Badea, K. B. Ghaghada, L. W. Hedlund, Y. Qi, H. Yuan, R. C. Bentley, and S. Mukundan, Jr. 2009. Micro-CT imaging of breast tumors in rodents using a liposomal, nanoparticle contrast agent. Int J Nanomedicine 4, 277-282.

Sasaki, M., S. Chubachi, N. Kameyama, M. Sato, M. Haraguchi, M. Miyazaki, S. Takahashi, and T. Betsuyaku. 2015. Evaluation of cigarette smoke-induced emphysema in mice using quantitative micro computed tomography. Am J Physiol Lung Cell Mol Physiol 308(10), L1039-1045. doi:10.1152/ ajplung.00366.2014.

Sawall, S., F. Bergner, R. Lapp, M. Mronz, M. Karolczak, A. Hess, and M. Kachelrieß. 2011. Low-dose cardio-respiratory phasecorrelated cone-beam micro-CT of small animals. Medical Physics 38(3), 1416-1424.

Sawall, S., J. Kuntz, M. Socher, M. Knaup, A. Hess, S. Bartling, and M. Kachelrie. 2012. Imaging of cardiac perfusion of free-breathing small animals using dynamic phase-correlated micro-CT. Medical Physics 39(12), 7499-7506.

Schambach, S. J., S. Bag, C. Groden, L. Schilling, and M. A. Brockmann. 2010. Vascular imaging in small rodents using micro-CT. Methods 50(1), 26-35. doi:10.1016/j.ymeth.2009.09.003.

Schirra, C.O., Pan, D., Roessl, E., Senpan, A., Schmieder, A.H., Scott, M., Lanza, G.M. 2012. Optimized ruptured plaque detection with ytterbium nanocolloids and spectral CT. Circulation 126 (supplement 1), Abstracts From the American Heart Association 2012 Scientific Sessions and Resuscitation Science Symposium, A13493-A13493.

Scotton, C. J., B. Hayes, R. Alexander, A. Datta, E. J. Forty, P. F. Mercer, A. Blanchard, and R. C. Chambers. 2013. Ex vivo micro-computed tomography analysis of bleomycin-induced lung fibrosis for preclinical drug evaluation. Eur Respir $J$ 42(6), 1633-1645. doi:10.1183/09031936.00182412.
Sheikh, A. Y., K. E. A. van der Bogt, T. C. Doyle, M. K. Sheikh, K. J. Ransohoff, Z. A. Ali, O. P. Palmer, R. C. Robbins, M. P. Fischbein, and J. C. Wu. 2010. Micro-CT for Characterization of Murine CV Disease Models. Jacc-Cardiovascular Imaging 3(7), 783-785. doi:10.1016/j.jcmg.2010.01.012.

Shofer, S., C. Badea, S. Auerbach, D. A. Schwartz, and G. A. Johnson. 2007. A micro-computed tomography-based method for the measurement of pulmonary compliance in healthy and bleomycin-exposed mice. Exp Lung Res 33(3-4), 169-183.

Shofer, S., C. Badea, Y. Qi, E. Potts, W. M. Foster, and G. A. Johnson. 2008. A micro-CT analysis of murine lung recruitment in bleomycin-induced lung injury. J Appl Physiol 105(2), 669-677. doi:10.1152/japplphysiol.00980.2007.

Song, J., Q. H. Liu, G. A. Johnson, and C. T. Badea. 2007. Sparseness prior based iterative image reconstruction for retrospectively gated cardiac micro-CT. Med Phys 34(11), 4476-4483.

Stolz, E., M. Yeniguen, M. Kreisel, M. Kampschulte, S. Doenges, D. Sedding, E. L. Ritman, T. Gerriets, and A. C. Langheinrich. 2011. Angioarchitectural changes in subacute cerebral venous thrombosis. A synchrotron-based micro- and nanoCT study. Neurolmage 54(3), 1881-1886. doi: 10.1016/j. neuroimage.2010.10.056.

Stutman, D., J. W. Stayman, M. Finkenthal, and J. H. Siewerdsen. 2013. High energy X-ray phase-contrast imaging using glancing angle grating interferometers. Proc. of SPIE 8668, Medical Imaging 2013: Physics of Medical Imaging 866814-1. March 19, 2013, doi:10.1117/12.2007930.

Taguchi, K. and J. S. Iwanczyk. 2013. Vision 20/20: Single photon counting X-ray detectors in medical imaging. Med Phys 40(10), 100901. doi:10.1118/1.4820371.

Taguchi, K. J. Xu, S. Srivastava, B. M. Tsui, J. Cammin, and Q. Tang. 2011. Interior region-of-interest reconstruction using a small, nearly piecewise constant subregion. Med Phys 38(3), 1307-1312.

Tapfer, A., M. Bech, A. Velroyen, J. Meiser, J. Mohr, M. Walter, J. Schulz, B. Pauwels, P. Bruyndonckx, and X. Liu. 2012. Experimental results from a preclinical X-ray phase-contrast CT scanner. Proceedings of the National Academy of Sciences 109(39), 15691-15696.

Tapfer, A., R. Braren, M. Bech, M. Willner, I. Zanette, T. Weitkamp, M. Trajkovic-Arsic, J. T. Siveke, M. Settles, and M. Aichler. 2013. X-ray phase-contrast CT of a pancreatic ductal adenocarcinoma mouse model. PloS One 8(3), e58439.

Tepel, M., P. Aspelin, and N. Lameire. 2006. Contrastinduced nephropathy: A clinical and evidence-based approach. Circulation 113(14), 1799-1806. doi:10.1161/ CIRCULATIONAHA.105.595090.

Torchilin, V. P., M. D. Frank-Kamenetsky, and G. L. Wolf. 1999. CT visualization of blood pool in rats by using long-circulating, iodine-containing micelles. Acad Radiol 6(1), 61-65.

Trubetskoy, V. S., G. S. Gazelle, G. L. Wolf, and V. P. Torchilin. 1997. Block-copolymer of polyethylene glycol and polylysine as a carrier of organic iodine: Design of long-circulating particulate contrast medium for X-ray computed tomography. $J$ Drug Target 4(6), 381-388. doi:10.3109/10611869709017895.

Tuy, H. K. 1983. An inversion formula for cone-beam reconstruction. SIAM J. Appl. Math 43, 546-562.

Ueno, T. et al. 2012. Non-invasive X-ray micro-computed tomographic evaluation of indomethacin on urethane-induced lung carcinogenesis in mice. Anticancer Res 32(11), 4773-4780. 
Umoh, J. U., A. V. Sampaio, I. Welch, V. Pitelka, H. A. Goldberg, T. M. Underhill, and D. W. Holdsworth. 2009. In vivo micro-CT analysis of bone remodeling in a rat calvarial defect model. Phys Med Biol 54(7), 2147-2161. doi:10.1088/0031-9155/54/7/020.

Vandeghinste, B., B. Trachet, M. Renard, C. Casteleyn, S. Staelens, B. Loeys, P. Segers, and S. Vandenberghe. 2011. Replacing vascular corrosion casting by in vivo micro-CT imaging for building 3D cardiovascular models in mice. Mol Imaging Biol 13(1), 78-86. doi:10.1007/s11307-010-0335-8.

Varenika, V., Y. Fu, J. J. Maher, D. Gao, S. Kakar, M. C. Cabarrus, and B. M. Yeh. 2013. Hepatic fibrosis: Evaluation with semiquantitative contrast-enhanced CT. Radiology 266(1), 151158. doi:10.1148/radiol.12112452.

von Smekal, L., M. Kachelriess, E. Stepina, and W. A. Kalender. 2004. Geometric misalignment and calibration in cone-beam tomography. Med Phys 31(12), 3242-3266.

Waarsing, J. H., J. S. Day, and H. Weinans. 2005. Longitudinal micro-CT scans to evaluate bone architecture. J Musculoskelet Neuronal Interact 5(4), 310-312.

Wang, C. L., R. H. Cohan, J. H. Ellis, S. Adusumilli, and N. R. Dunnick. 2007. Frequency, management, and outcome of extravasation of nonionic iodinated contrast medium in 69,657 intravenous injections. Radiology 243(1), 80-87. doi:10.1148/ radiol.2431060554.

Wang, H., L. Zheng, C. Peng, M. Shen, X. Shi, and G. Zhang. 2013. Folic acid-modified dendrimer-entrapped gold nanoparticles as nanoprobes for targeted CT imaging of human lung adencarcinoma. Biomaterials 34(2), 470-480. doi:10.1016/j. biomaterials.2012.09.054.

Wathen, C. A., N. Foje, T. van Avermaete, B. Miramontes, S. E. Chapaman, T. A. Sasser, R. Kannan, S. Gerstler, and W. M. Leevy. 2013. In vivo X-ray computed tomographic imaging of soft tissue with native, intravenous, or oral contrast. Sensors (Basel) 13(6), 6957-6980. doi:10.3390/s130606957.

Wetzel, A.W., C. T. Badea, S. M. Pomerantz, N. Mistry, D. Nave, and G. A. Johnson. 2007. Measurement and modeling of 4D live mouse heart volumes from $\mathrm{CT}$ time series. Proceedings of SPIE 6491, 64910J.

Wicklein, J., H. Kunze, W. A. Kalender, and Y. Kyriakou. 2012. Image features for misalignment correction in medical flat-detector CT. Med Phys 39(8), 4918-4931. doi:10.1118/1.4736532.

Willner, M., J. Herzen, S. Grandl, S. Auweter, D. Mayr, A. Hipp, M. Chabior, A. Sarapata, K. Achterhold, and I. Zanette. 2014. Quantitative breast tissue characterization using gratingbased X-ray phase-contrast imaging. Physics in Medicine and Biology 59(7), 1557.

Wilson, P. D. 2008. Mouse models of polycystic kidney disease. Curr Top Dev Biol 84:311-350. doi:10.1016/S0070-2153(08) 00606-6.

Winter, P. M., H. P. Shukla, S. D. Caruthers, M. J. Scott, R. W. Fuhrhop, J. D. Robertson, P. J. Gaffney, S. A. Wickline, and G. M. Lanza. 2005. Molecular imaging of human thrombus with computed tomography. Acad Radiol 12(Suppl 1), S9-S13.

Wolfe, T., D. Chatterjee, J. Lee, J. D. Grant, S. Bhattarai, R. Tailor, G. Goodrich, P. Nicolucci, and S. Krishnan. 2015. Targeted gold nanoparticles enhance sensitization of prostate tumors to megavoltage radiation therapy in vivo. Nanomedicine 11(5):1277-83. July 2015. Corrected article 12(3):851-52. April 2016. doi:10.1016/j.nano.2014.12.016.

Wyss, C., S. C. Schaefer, L. Juillerat-Jeanneret, L. Lagopoulos, H. A. Lehr, C. D. Becker, and X. Montet. 2009. Molecular imaging by micro-CT: Specific E-selectin imaging. Eur Radiol 19(10), 2487-2494. doi:10.1007/s00330-009-1434-2.

Xu, F. and K. Mueller. 2007. Real-time 3D computed tomographic reconstruction using commodity graphics hardware. Phys Med Biol 52(12), 3405-3419. doi:10.1088/0031-9155/52/12/006.

Xu, R., F. Franchi, B. Miller, J. A. Crane, K. M. Peterson, P. J. Psaltis, P. C. Harris, L. O. Lerman, and M. Rodriguez-Porcel. 2013. Polycystic kidneys have decreased vascular density: A micro-CT study. Microcirculation 20(2), 183-189. doi:10.1111/micc.12022.

Xue, S. H., Y. Wang, M. X. Wang, L. Zhang, X. X. Du, H. C. Gu, and C. F. Zhang. 2014. Iodinated oil-loaded, fluorescent mesoporous silica-coated iron oxide nanoparticles for magnetic resonance imaging/computed tomography/fluorescence trimodal imaging. International Journal of Nanomedicine 9, 2527-2538. doi:10.2147/Ijn.S59754.

Yang, K., A. L. Kwan, D. F. Miller, and J. M. Boone. 2006. A geometric calibration method for cone beam CT systems. Med Phys 33(6), 1695-1706.

Yao, W., T. Hadi, Y. Jiang, J. Lotz, T. J. Wronski, and N. E. Lane. 2005. Basic fibroblast growth factor improves trabecular bone connectivity and bone strength in the lumbar vertebral body of osteopenic rats. Osteoporos Int 16(12), 1939-1947. doi:10.1007/s00198-005-1969-2.

Yu, H. et al. 2012. Medipix-based spectral micro-CT. CT Li Lun Yu Ying Yong Yan Jiu 21(4), 583.

Yu, S. B. and A. D. Watson. 1999. Metal-based X-ray contrast media. Chem Rev 99(9), 2353-2378.

Yue, G.Z., Q. Qiu, B. Gao, Y. Cheng, J. Zhang, H. Shimoda, S. Chang, J. P. Lu, and O. Zhou. 2002. Generation of continuous and pulsed diagnostic imaging X-ray radiation using a carbon-nanotube-based field-emission cathode. Applied Physics Letters 81(2), 355-357.

Zhao, X., J. Bian, E. Y. Sidky, S. Cho, P. Zhang, X. Pan, and IEEE. 2007. GPU-based 3D cone-beam CT image reconstruction: Application to micro CT. In 2007 IEEE Nuclear Science Symposium Conference Record, Vols 1-11, 3922-3925.

Zheng, J., D. Jaffray, and C. Allen. 2009. Quantitative CT imaging of the spatial and temporal distribution of liposomes in a rabbit tumor model. Mol Pharm 6(2), 571-580. doi:10.1021/mp800234r.

Zheng, J., G. Perkins, A. Kirilova, C. Allen, and D. A. Jaffray. 2006. Multimodal contrast agent for combined computed tomography and magnetic resonance imaging applications. Invest Radiol 41(3), 339-348.

Zhou, Y., H. Chen, N. Ambalavanan, G. Liu, V. B. Antony, Q. Ding, H. Nath, J. F. Eary, and V. J. Thannickal. 2015. Noninvasive Imaging of Experimental Lung Fibrosis. Am J Respir Cell Mol Biol 53(1), 8-13. doi:10.1165/rcmb.2015-0032TR.

Zhu, J. Y., L. F. Zheng, S. H. Wen, Y. Q. Tang, M. W. Shen, G. X. Zhang, and X. Y. Shi. 2014. Targeted cancer theranostics using alpha-tocopheryl succinate-conjugated multifunctional dendrimer-entrapped gold nanoparticles. Biomaterials 35(26), 7635-7646. doi:10.1016/j.biomaterials.2014.05.046. 